Interfaces and Free Boundaries 13 (2011), 29-55

DOI 10.4171/IFB/247

\title{
Phase field approximation of a kinetic moving-boundary problem modelling dissolution and precipitation
}

\author{
T. L. VAN NOORDEN \\ Department of Mathematics and Computer Science, Technische Universiteit Eindhoven, \\ PO Box 513, 5600 MB Eindhoven, The Netherlands \\ E-mail:T.L.v.Noorden@tue.nl \\ C. ECK \\ Institut für Angewandte Analysis und Numerische Simulation, \\ Fachbereich Mathematik, Universität Stuttgart, \\ Pfaffenwaldring 57, D-70569 Stuttgart, Germany \\ E-mail: Christof.Eck@mathematik.uni-stuttgart.de
}

[Received 15 March 2009 and in revised form 19 November 2010]

\begin{abstract}
We present a phase field model which approximates a one-phase Stefan-like problem with a kinetic condition at the moving boundary, and which models a dissolution and precipitation reaction. The concentration of dissolved particles is variable on one side of the free boundary and jumps across the free boundary to a fixed value given by the constant concentration of the particles in the precipitate. Using a formal asymptotic analysis we show that the phase field model approximates the appropriate sharp interface limit. The existence and uniqueness of solutions to the phase field model is studied. By numerical experiments the approximating behaviour of the phase field model is investigated.
\end{abstract}

\section{Introduction}

In this paper we introduce a phase field model describing a one-phase free boundary problem with a kinetic condition at the moving boundary. The motivating background is the modelling of a process involving transport of solutes by diffusion in a fluid undergoing a precipitation/dissolution reaction. This process can be modelled by a sharp interface free boundary problem as presented in [16]. The model in [16] takes into account the dissolved particles present in an aqueous solution. In a precipitation reaction a fixed number of dissolved particles can precipitate in the form of one particle of a (crystalline) solid, which is attached to the boundary of the region occupied by the fluid, and which is immobile. Also the reverse reaction of dissolution is possible. In the case of dissolution of solid particles the boundary of the fluid region recedes, and in the case of precipitation it advances.

The phase field model presented in this work is intended to model the same precipitation/dissolution reaction. Using a formal asymptotic analysis we show that the phase field model approximates in the limit $\epsilon \rightarrow 0$ the sharp interface model presented in [16]. Here $\epsilon$ is the thickness of an interfacial layer containing the moving boundary [6]. Phase field models are widely used in the modelling of phase transitions and solidification processes [2, 3, 5, 6, 7] that can be described using a Stefan problem with kinetic and curvature undercooling. In this case usually only the jump of the internal energy (the latent heat) or of the concentration (miscibility gap) across the interface can be prescribed. In the case of precipitation and dissolution the concentration also jumps across the interface, but instead of the magnitude of the jump, the value of the concentration on one side of the 
interface is prescribed while leaving it variable on the other side. The fixed value of the concentration on one side of the interface is given by the fixed and constant concentration of particles in the precipitate. To achieve this, we need a singular coefficient in the diffusion equation of the phase field model, or a coefficient that becomes singular in the limit $\epsilon \rightarrow 0$.

The reason for proposing and studying such a phase field formulation of the crystal precipitation/dissolution process is the same as for the classical Stefan problem and other sharp interface problems: the theoretical mathematical analysis for the phase field problem is usually much more straightforward for the phase field formulation than for the sharp interface problem. The phase field formulation is usually also more easy to deal with numerically and in addition rigorous homogenization results can be obtained (see, for example, [9]).

We propose a phase field model for the (mass per volume) concentration of dissolved particles $u(t, x)$ and the phase parameter $\varphi(t, x)$, which has the form

$$
\begin{aligned}
\alpha \epsilon^{2} \partial_{t} \varphi-\epsilon^{2} \Delta \varphi+p^{\prime}(\varphi)+\epsilon \beta k^{\prime}(\varphi)\left(f(u)+(u-\rho) f^{\prime}(u)\right) & =0, \\
\partial_{t} u-D \nabla \cdot\left(\nabla u+(\rho-u) \frac{k^{\prime}(\varphi)}{k(\varphi)} \nabla \varphi\right) & =0,
\end{aligned}
$$

where $p(\varphi)$ is a double well potential, $f(u)$ is a rate function, $k(\varphi)$ is an interpolation function that decreases monotonically from 1 to 0 for $\varphi$ ranging from -1 to 1 , with vanishing first derivative at $\varphi= \pm 1$. The physical parameters $\alpha, \beta, D$ and $\rho$ represent the relaxation time, the reciprocal surface tension, the diffusion coefficient and the fixed (mass per volume) concentration of particles in the crystalline solid, respectively. The region where $\varphi \approx-1$ corresponds to the fluid region and the region where $\varphi \approx 1$ corresponds to the crystalline solid, and $\varphi$ should have a steep transition from -1 to 1 at the position of the interface between the fluid and solid. As $k(\varphi)$ vanishes for $\varphi=1$, the coefficient $k^{\prime}(\varphi) / k(\varphi)$ is singular at $\varphi=1$. In the asymptotic analysis we will see that this enforces $u \approx \rho$ when $\varphi \approx 1$ to leading order. This mechanism implements the requirement that $u$ has a fixed value on the side of the interface that represents the crystalline solid.

The paper is organized as follows. In Section 2 we briefly discuss the sharp interface problem that is approximated by the proposed phase field model. In Section 3 the phase field model is introduced, and in Section 4 we show by a formal asymptotic expansion that the phase field model approximates the sharp interface problem discussed in Section 2 . We prove the solvability of our phase field model in Section 5, and in Section 6 we illustrate numerically that solutions to the phase field model approximate solutions to the sharp interface equations for small $\epsilon$.

\section{The sharp interface model}

For the formulation of the sharp interface model, we consider a domain $\Omega \subset \mathbb{R}^{d}$ having a sufficiently regular boundary $\Gamma$. We assume that $\Omega$ contains two distinct phases: fluid and solid, which occupy two disjoint time-dependent a priori unknown regions $\Omega_{f}(t)$ and $\Omega_{s}(t)$, respectively. These regions are separated by the moving interface $\Gamma_{I}(t)$, where the precipitation/dissolution reaction is taking place. We have $\Omega:=\Omega_{f}(t) \cup \Omega_{s}(t) \cup \Gamma_{I}(t)$. Furthermore, the boundary of $\Omega$ is divided into $\Gamma_{f}(t)$ and $\Gamma_{s}(t)$, where $\Gamma_{i}(t):=\partial \Omega \cap \partial \Omega_{i}(t)$ for $i=f, s$. We denote by $n$ the normal vector to $\partial \Omega_{f}(t)$ pointing outward, which means that on $\Gamma_{I}(t)$ the vector $n$ points into the solid part.

The fluid in $\Omega_{f}(t)$ contains dissolved solutes which precipitate on the wet part of the grain boundary and form a crystalline solid. Generally the dissolved particles, or solutes, consist of anions 
and cations. The ratio between their concentrations may vary within the fluid, but in the precipitate a fixed number of ions, say $n_{1}$, and fixed number of cations, say $n_{2}$, form together one particle of the crystalline solid. We restrict ourselves to the situation where also in the fluid the ratio between the ion and cation concentrations is constant and equal to the ratio of their concentrations in the crystalline solid, i.e. $n_{1} / n_{2}$. One can show that if this is initially the case, and if one assumes that the diffusion coefficients for the ions and cations are the same, and in the absence of electric forces and fields, the ratio of the concentrations will stay equal to $n_{1} / n_{2}$ in the fluid [11, 13, 15, 16]. This means that under these assumptions we may restrict our attention to the concentration $u$ of one solute species (the cation). Also note that we do not take into account volume changes due to the precipitation/dissolution reaction. This would require taking into account fluid flow with either a time dependent domain $\Omega$ or an in/out-flow boundary condition [14]. At the moment we neglect these volume changes and we assume that there is no fluid flow and that the only means of transport of the solute in the fluid phase is diffusion. This restricts our model, but the model works well when the volume changes are small, or when transport by diffusion dominates transport by fluid flow.

The solid domain $\Omega_{s}(t)$ is occupied by the crystalline solid in which the ions have a fixed, constant (mass per volume) concentration, denoted by $\rho$.

All the quantities and equations involved are considered dimensionless. For $T>0$ we define

$$
\begin{array}{rlrl}
J & :=(0, T], & \\
Q(T) & :=J \times \Omega, & \\
Q_{i}(T) & :=\left\{(t, x) \mid t \in J, x \in \Omega_{i}(t)\right\} & & \text { with } i=f, s, \\
G_{i}(T) & :=\left\{(t, x) \mid t \in J, x \in \Gamma_{i}(t)\right\} & & \text { with } i=f, s, I .
\end{array}
$$

Then the model equations are given by

$$
\left\{\begin{aligned}
\partial_{t} u & =D \Delta u & & \text { in } Q_{f}(T), \\
\nabla u \cdot n & =0 & & \text { on } G_{f}(T), \\
v_{n}(\rho-u) & =D(\nabla u \cdot n) & & \text { on } G_{I}(T) \cap Q(T), \\
u(0, x) & =u_{0}(x) & & \text { on } \Omega_{f}(0),
\end{aligned}\right.
$$

where $D$ is the diffusion coefficient of the solute, $v_{n}$ denotes the normal velocity of $\Gamma_{I}(t)$, and $n$ is, as introduced above, the outward pointing unit normal. Equation 43 ) is a Rankine-Hugoniot condition [10] and ensures the conservation of mass. The kinetic condition for the normal velocity $v_{n}$ of the interface $\Gamma_{I}(t)$ reads

$$
v_{n}+\sigma \kappa=-f(u) \quad \text { on } G_{I}(T) \cap Q(T),
$$

where $\sigma$ is the surface tension and $\kappa$ is the curvature of the interface $\Gamma_{I}(t)$. Note that the equation (5) (just as $(43)$ ) holds only in the interior of $\Omega$. In order to make sure that the moving boundary does not leave the domain $\Omega$, we impose the condition

$$
v_{n}=[-\sigma \kappa-f(u)]_{-} \quad \text { on } G_{I}(T) \cap G(T),
$$

with $[x]_{-}=\min \{0, x\}$, and where $G(T)$ is the lateral boundary $G(T):=J \times \Gamma$. We refer to [16] for the exact structure of the rate function $f(u)$ in the specific case of dissolution and precipitation, and also for a well-posedness result for the equations (4)-(5) in a one-dimensional setting. Here we only mention that $f(u)$ is Lipschitz and non-decreasing. The function $u_{0}$ appearing in the initial condition, as well as the initial fluid domain configuration $\Omega_{f}(0)$, are assumed to be given. 
The term $\sigma \kappa$ in (5) modelling curvature effects does not of course appear in one-dimensional settings, such as, for example, in [16]. Furthermore, surface tension is in general anisotropic due to the crystalline structure of the solid material. However, in the present paper we restrict ourselves to isotropic surface tension.

\section{The phase field model}

In Section 4 we show by a formal asymptotic analysis that solutions to the phase field model (1)-(2) approximate solutions to the sharp interface model discussed in the previous section. In fact, we present a formal asymptotic analysis of the phase field model with a regularized version of (2): we replace the fraction $k^{\prime}(\varphi) / k(\varphi)$ in (2), which has a singularity for $\varphi=1$, by the fraction $k^{\prime}(\varphi) /(k(\varphi)+\epsilon)$, which remains bounded for all $\varphi$. The regularized version is also used for the numerical computations discussed in Section 6 Since the asymptotic analysis presented in Section 4 does, with slight variations, also apply for (1)-(2), both versions of the phase field model approximate the same sharp interface model. In Section 5] we prove existence and uniqueness of solutions to the regularized phase field model as well as the existence of solutions to the singular version (1)-2) when the initial condition $\varphi_{0}$ satisfies the condition $\ln \left(1-\varphi_{0}\right) \in H^{1}(\Omega)$.

To approximate the sharp interface problem (4)-(5) we consider the following phase field model:

$$
\begin{aligned}
\alpha \epsilon^{2} \partial_{t} \varphi-\epsilon^{2} \Delta \varphi+p^{\prime}(\varphi)+\epsilon \beta k^{\prime}(\varphi)\left(f(u)+(u-\rho) f^{\prime}(u)\right) & =0, \\
\partial_{t} u-D \nabla \cdot\left(\nabla u+(\rho-u) \frac{k^{\prime}(\varphi)}{k(\varphi)+\epsilon} \nabla \varphi\right) & =0
\end{aligned}
$$

on the time-space cylinder $Q(T)$. The partial differential equations $(7)-(8)$ are complemented by the initial data

$$
\begin{array}{ll}
\varphi(0, x)=\varphi_{0}(x) & \text { for all } x \in \Omega, \\
u(0, x)=u_{0}(x) & \text { for all } x \in \Omega,
\end{array}
$$

and homogeneous Neumann conditions on the lateral boundary $G(T)$,

$$
\begin{aligned}
& \nabla \varphi \cdot n=0, \\
& \nabla u \cdot n=0 .
\end{aligned}
$$

There is some freedom in choosing the exact form of the functions $k$ and $p$. A typical choice for the function $p$ is the double well potential given by

$$
p(\varphi):=\frac{1}{2}\left(\varphi^{2}-1\right)^{2} .
$$

In this paper, however, we need a potential $p$ with slightly different properties, and we will use the potential given by

$$
p(\varphi):=\frac{1}{2}\left(\varphi^{2}-1\right)^{4} .
$$

The reasons for choosing this potential will become clear later on (see the remarks at the end of 
Section 4.3). The function $k(\varphi)$ should interpolate between 1 and 0 for $\varphi$ ranging from -1 to 1 , and have vanishing first derivative for $\varphi= \pm 1$. In this paper we define $k(\varphi)$ by

$$
k(\varphi):= \begin{cases}1 & \text { for } \varphi<-1, \\ 1-\frac{1}{2}(1+\varphi)^{2} & \text { for }-1 \leqslant \varphi \leqslant 0, \\ \frac{1}{2}(1-\varphi)^{2} & \text { for } 0<\varphi \leqslant 1, \\ 0 & \text { for } \varphi>1\end{cases}
$$

\section{Formal asymptotic analysis for $\epsilon \rightarrow 0$}

In this section we present a formal asymptotic analysis of the phase field model (7)- 87 with $p$ and $k$ given by (14) and (15). In order to describe the transition from the phase field model to the sharp interface model we use the matched asymptotic expansion. This expansion consists of two parts, an outer expansion valid far away from the free boundary, and an inner expansion valid close to the free boundary. The outer expansions for $u$ and $\varphi$ are given by

$$
\begin{aligned}
& u(t, x)=U_{0}(t, x)+\epsilon U_{1}(t, x)+\epsilon^{2} U_{2}(t, x)+\cdots, \\
& \varphi(t, x)=\Phi_{0}(t, x)+\epsilon \Phi_{1}(t, x)+\epsilon^{2} \Phi_{2}(t, x)+\cdots .
\end{aligned}
$$

For the inner expansion we use a transform of variables into local curvilinear coordinates [4] that are derived from the zero-level set $\Gamma_{I, \epsilon}(t):=\left\{x \in \mathbb{R}^{d} \mid \varphi(t, x)=0\right\}$ of the solution $\varphi$ to the phase field model with parameter $\epsilon$. We assume that this level set is sufficiently smooth and smoothly approaches the moving interface $\Gamma_{I}(t)$ as $\epsilon \rightarrow 0$. For a point $x \in \Omega$, let $r(t, x)$ denote the signed distance of $x$ to $\Gamma_{I, \epsilon}(t)$ with negative $r(t, x)$ for $x$ in the liquid domain, and let $s \mapsto y_{\epsilon}(t, s)$ for $s \in P \subset \mathbb{R}^{d-1}$ be an orthogonal parametrization of $\Gamma_{I, \epsilon}(t)$. Then any point in a neighbourhood of $\Gamma_{I, \epsilon}(t)$ can be represented by

$$
x=y_{\epsilon}(t, s)+r n_{\epsilon}(t, s),
$$

where $n_{\epsilon}(t, s)$ is the normal vector to $\Gamma_{I, \epsilon}(t)$ at $y_{\epsilon}(t, s)$. The corresponding coordinates $r=r(t, x)$ and $s=s(t, x)$ satisfy

$$
\begin{aligned}
& |\nabla r|^{2}=1, \quad \nabla r \cdot \nabla s=0, \\
& \partial_{t} r=-v_{n}, \\
& \Delta r=\frac{\kappa+2 \Pi r}{1+\kappa r+\Pi r^{2}},
\end{aligned}
$$

with $\kappa$ and $\Pi$ denoting the mean and Gaussian curvatures of $\Gamma_{I, \epsilon}$ and $v_{n}$ denoting the normal velocity of $\Gamma_{I, \epsilon}$. The normal is pointing outward from the liquid domain. Furthermore we assume that the curvature and the normal velocity satisfy the expansions

$$
\kappa=\kappa_{0}+O(\epsilon) \text { and } v_{n}=v_{n, 0}+O(\epsilon),
$$

where $\kappa_{0}$ and $v_{n, 0}$ are the curvature and the velocity of the moving interface $\Gamma_{I}(t)$. Using the transformations $\tilde{u}(t, r(t, x), s(t, x))=u(t, x)$ and $\tilde{\varphi}(t, r(t, x), s(t, x))=\varphi(t, x)$, we transform 
the differential operators involved into (see, for example, [5])

$$
\begin{aligned}
& \partial_{t} u=\left(\partial_{t}-v_{n} \partial_{r}+\partial_{t} s \cdot \nabla_{s}\right) \tilde{u}, \\
& \partial_{t} \varphi=\left(\partial_{t}-v_{n} \partial_{r}+\partial_{t} s \cdot \nabla_{s}\right) \tilde{\varphi}, \\
& \Delta u=\left(\partial_{r}^{2}+\Delta r \partial_{r}+\Delta s \cdot \nabla_{s}+\sum_{i=1}^{d-1}\left|\nabla s_{i}\right|^{2} \partial_{s_{i}}^{2}\right) \tilde{u}, \\
& \nabla \cdot(K \nabla \varphi)=\left(\partial_{r}\left(K \partial_{r}\right)+K \Delta r \partial_{r}+K \Delta s \cdot \nabla_{s}+\sum_{i=1}^{d-1}\left|\nabla s_{i}\right|^{2} \partial_{s_{i}}\left(K \partial_{s_{i}}\right)\right) \tilde{\varphi},
\end{aligned}
$$

where $K=(\rho-u) k^{\prime}(\varphi) /(k(\varphi)+\epsilon)$.

Close to the moving boundary $\Gamma_{I, \epsilon}$, the solution to the phase field model is represented in the new coordinates $(t, r, s)$. In the limit $\epsilon \rightarrow 0$, we expect the diffusive layer to shrink to a sharp interface positioned in $\Gamma_{I}(t)$. We perform the further change of coordinate $\xi=r / \epsilon$, introduce the variables

$$
\hat{u}(t, \xi, s)=\hat{u}\left(t, \frac{r}{\epsilon}, s\right)=\tilde{u}(t, r, s) \quad \text { and } \quad \phi(t, \xi, s)=\phi\left(t, \frac{r}{\epsilon}, s\right)=\tilde{\varphi}(t, r, s),
$$

and use the following expansion ansatz:

$$
\begin{aligned}
& \hat{u}(t, \xi, s)=u_{0}(t, \xi, s)+\epsilon u_{1}(t, \xi, s)+\epsilon^{2} u_{2}(t, \xi, s)+\cdots \\
& \phi(t, \xi, s)=\phi_{0}(t, \xi, s)+\epsilon \phi_{1}(t, \xi, s)+\epsilon^{2} \phi_{2}(t, \xi, s)+\cdots .
\end{aligned}
$$

For the equations for the inner expansions we also need to transform and expand the differential operators. The asymptotic expansions of the differential operators represented in the coordinates $(t, \xi, s)$ can be written as

$$
\begin{aligned}
& \partial_{t}=-\epsilon^{-1} v_{n} \partial_{\xi}+\left(\partial_{t}+\partial_{t} s \cdot \nabla_{s}\right), \\
& \Delta=\epsilon^{-2} \partial_{\xi}^{2}+\epsilon^{-1} \Delta r \partial_{\xi}+\left(\Delta s \cdot \nabla_{s}+\sum_{i=1}^{d-1}\left|\nabla s_{i}\right|^{2} \partial_{s_{i}}^{2}\right), \\
& \nabla \cdot(K \nabla)=\epsilon^{-2} \partial_{\xi}\left(K \partial_{\xi}\right)+\epsilon^{-1} \kappa_{0} K \partial_{\xi}+O(1) .
\end{aligned}
$$

\subsection{Matching conditions}

In an intermediate region of distance proportional to $\sqrt{\epsilon}$ the inner and outer expansions describe the same function. This is realized by matching conditions for both expansions. We write the outer expansion in terms of the local variables $(t, \xi, s)$ using (16) with $r=\epsilon \xi$. The normal vector can be expanded in a series

$$
n_{\epsilon}(t, s)=n_{0}(t, s)+\epsilon n_{1}(t, s)+\cdots .
$$

The point $y_{\epsilon}$ admits the expansion

$$
y_{\epsilon}(t, s)=y_{0}(t, s)+\epsilon n_{0}(t, s) y_{1}(t, s)+\cdots .
$$

The matching conditions are derived from

$$
\begin{aligned}
& u\left(t, y_{\epsilon}+\epsilon \xi n_{\epsilon}\right)=U_{0}\left(t, y_{0 \pm}\right)+\epsilon\left(U_{1}\left(t, y_{0 \pm}\right)+\left(\nabla U_{0}\left(t, y_{0 \pm}\right) \cdot n_{0}\right)\left(y_{1}+\xi\right)\right)+\cdots \\
& \varphi\left(t, y_{\epsilon}+\epsilon \xi n_{\epsilon}\right)=\Phi_{0}\left(t, y_{0 \pm}\right)+\epsilon\left(\Phi_{1}\left(t, y_{0 \pm}\right)+\left(\nabla \Phi_{0}\left(t, y_{0 \pm}\right) \cdot n_{0}\right)\left(y_{1}+\xi\right)\right)+\cdots
\end{aligned}
$$


where the index \pm at $y_{0}$ indicates the limit at the free boundary from the "fluid" $(\xi<0)$ or the "solid" $(\xi>0)$ side. This distinction is necessary, since the functions $U_{i}$ and $\Phi_{i}$ may be discontinuous at the free boundary. Now we match the inner and outer expansions by equating

$$
\begin{aligned}
& \hat{u}(t, \xi, s)=u\left(t, y_{\epsilon}(t, s)+\epsilon \xi n_{\epsilon}(t, s)\right), \\
& \phi(t, \xi, s)=\varphi\left(t, y_{\epsilon}(t, s)+\epsilon \xi n_{\epsilon}(t, s)\right) .
\end{aligned}
$$

Next we substitute the inner expansions $177-18$ and the expansions $190-(20)$ in the equations above, we take the limit $\xi \rightarrow \pm \infty$ and collect the terms of different order in $\epsilon$. In this way we obtain, for the terms of order $\epsilon^{0}$,

$$
\begin{aligned}
& \lim _{\xi \rightarrow \pm \infty} u_{0}(t, \xi, s)=U_{0}\left(t, y_{0 \pm}(t, s)\right), \\
& \lim _{\xi \rightarrow \pm \infty} \phi_{0}(t, \xi, s)=\Phi_{0}\left(t, y_{0 \pm}(t, s)\right) .
\end{aligned}
$$

For the terms of order $\epsilon^{1}$, we obtain

$$
\begin{aligned}
& \lim _{\xi \rightarrow \pm \infty}\left[u_{1}(t, \xi, s)-U_{1}\left(t, y_{0 \pm}(t, s)\right)-\left(\nabla U_{0}\left(t, y_{0 \pm}(t, s)\right) \cdot n_{0}(t, s)\right)\left(y_{1}(t, s)+\xi\right)\right]=0, \\
& \lim _{\xi \rightarrow \pm \infty}\left[\phi_{1}(t, \xi, s)-\Phi_{1}\left(t, y_{0 \pm}(t, s)\right)-\left(\nabla \Phi_{0}\left(t, y_{0 \pm}(t, s)\right) \cdot n_{0}(t, s)\right)\left(y_{1}(t, s)+\xi\right)\right]=0 .
\end{aligned}
$$

In a similar way we obtain matching conditions for the derivatives with respect to $\xi$ : we equate

$$
\begin{aligned}
\partial_{\xi} \hat{u}(t, \xi, s) & =\epsilon \nabla u\left(t, y_{\epsilon}(t, s)+\epsilon \xi n_{\epsilon}(t, s)\right) \cdot n_{\epsilon}(t, s), \\
\partial_{\xi} \phi(t, \xi, s) & =\epsilon \nabla \varphi\left(t, y_{\epsilon}(t, s)+\epsilon \xi n_{\epsilon}(t, s)\right) \cdot n_{\epsilon}(t, s),
\end{aligned}
$$

substitute the expansions, take the limit $\xi \rightarrow \pm \infty$, and obtain, for the terms of order $\epsilon^{0}$,

$$
\begin{aligned}
& \lim _{\xi \rightarrow \pm \infty} \partial_{\xi} u_{0}=0, \\
& \lim _{\xi \rightarrow \pm \infty} \partial_{\xi} \phi_{0}=0,
\end{aligned}
$$

and for the terms of order $\epsilon^{1}$,

$$
\begin{aligned}
& \lim _{\xi \rightarrow \pm \infty} \partial_{\xi} u_{1}(t, \xi, s)=\nabla U_{0}\left(t, y_{0 \pm}(t, s)\right) \cdot n_{0}(t, s), \\
& \lim _{\xi \rightarrow \pm \infty} \partial_{\xi} \phi_{1}(t, \xi, s)=\nabla \Phi_{0}\left(t, y_{0 \pm}(t, s)\right) \cdot n_{0}(t, s) .
\end{aligned}
$$

\subsection{Outer expansion}

By substituting the outer expansions in the differential equations, we obtain a sequence of problems. For the outer expansion, however, we will only look at the $O\left(\epsilon^{0}\right)$ problem. First we concentrate on the phase field equation. By the term of order $\epsilon^{0}$ in the phase field equation, we have $p^{\prime}\left(\Phi_{0}\right)=0$, from which it follows that

$$
\Phi_{0}= \pm 1 \quad \text { a.e. in } \Omega \text {. }
$$


The remaining zero $\Phi_{0}=0$ of $p^{\prime}$ is not considered, because it is unstable due to $p^{\prime \prime}\left(\Phi_{0}\right)<0$. The function $\Phi_{0}$ represents the limit of the solutions $\varphi_{\epsilon}$ to (7) as $\epsilon \rightarrow 0$, and unstable stationary solutions cannot be reached by this limit. This can be formally seen from the linearized equation

$$
\alpha \epsilon^{2} \partial_{t} \varphi_{\epsilon}=-p^{\prime \prime}\left(\Phi_{0}\right)\left(\varphi_{\epsilon}-\Phi_{0}\right)+\mathcal{O}(\epsilon)
$$

If $\epsilon$ is small, then $p^{\prime \prime}\left(\Phi_{0}\right)$ dominates the right hand side. For $p^{\prime \prime}\left(\Phi_{0}\right)<0$ any deviation from $\Phi_{0}$ will become larger with increasing time.

For $\Phi_{0}=-1$, we expand $k^{\prime}(\varphi) /(k(\varphi)+\epsilon)$ around $\varphi=-1$ and collect the $\epsilon^{0}$ terms in the diffusion equation to obtain

$$
\partial_{t} U_{0}-D \Delta U_{0}=0 \quad \text { when } \Phi_{0}=-1
$$

For $\Phi_{0}=1$, we need to be more careful and look in more detail at the term $\frac{k^{\prime}(\varphi)}{k(\varphi)+\epsilon} \nabla \varphi$. We use the definition 15] of $k(\varphi)$ near $\varphi=1$, we substitute the outer expansion and we use $\Phi_{0}=1$ and $\nabla \Phi_{0}=0$ so that we have

$$
\begin{aligned}
\frac{\varphi-1}{\frac{1}{2}(1-\varphi)^{2}+\epsilon} \nabla \varphi & =\frac{\epsilon \Phi_{1}+\epsilon^{2}(\ldots)}{\frac{1}{2}\left(\epsilon \Phi_{1}+\epsilon^{2}(\ldots)\right)^{2}+\epsilon}\left(\epsilon \nabla \Phi_{1}+\epsilon^{2}(\ldots)\right) \\
& =\frac{\Phi_{1}+\epsilon(\ldots)}{\epsilon \frac{1}{2}\left(\Phi_{1}+\epsilon(\ldots)\right)^{2}+1}\left(\epsilon \nabla \Phi_{1}+\epsilon^{2}(\ldots)\right) .
\end{aligned}
$$

We conclude that the term $\frac{k^{\prime}(\varphi)}{k(\varphi)+\epsilon} \nabla \varphi$ is $O(\epsilon)$ when $\Phi_{0}=1$. This means that if we collect the $\epsilon^{0}$ terms in the diffusion equation we also get

$$
\partial_{t} U_{0}-D \Delta U_{0}=0 \quad \text { when } \Phi_{0}=1
$$

\subsection{Inner expansion}

Now we substitute the inner expansions of the differential operators and those of $\hat{u}$ and $\phi$ into the differential equations and collect terms of the same order in $\epsilon$. In addition we multiply the diffusion equation with $\epsilon^{2}$ so that the lowest order terms in both equations are of order $\epsilon^{0}$. Then we again obtain a sequence of problems.

The first problem consists of the terms of order $\epsilon^{0}$ in both the diffusion equation and the phase field equation. Let us first consider the equation for $\phi_{0}$ :

$$
-\partial_{\xi}^{2} \phi_{0}+p^{\prime}\left(\phi_{0}\right)=0
$$

This equation is supplemented by boundary conditions at $\pm \infty$, derived from the matching condition 22):

$$
\lim _{\xi \rightarrow \pm \infty} \phi_{0}(t, \xi, s)= \pm 1
$$

Due to the condition $\phi(t, 0, s)=0$ and the fact that $\Gamma_{I, \epsilon}(t)$ is supposed to converge to $\Gamma_{I}(t)$ for $\epsilon \rightarrow 0$ we also have $\phi_{0}(t, 0, s)=0$. Since $p$ is an even function, i.e. $p(x)=p(-x)$ (see (14)), and 
nonnegative, we can reduce (32) together with the conditions $\phi_{0}(t, 0, s)=0$ and $\phi_{0}(t, \xi, s) \rightarrow 1$ as $\xi \rightarrow \infty$ to the first order equation

$$
\partial_{\xi} \phi_{0}=\sqrt{2 p\left(\phi_{0}\right)}, \quad \phi_{0}(0)=0 .
$$

This equation has, with the given choice for the double well potential, a unique, strictly increasing solution, independent of $t$ and $s$, which we from now on denote by $\phi_{0}(\xi)$.

Now we turn to the equation for $u_{0}$. For $\xi \leqslant \bar{\xi}$, with $\bar{\xi} \in \mathbb{R}$ a constant, we have $\phi_{0}(\xi) \leqslant \phi_{0}(\bar{\xi})$ $<1$. This means that for $\xi \leqslant \bar{\xi}$ we have the expansion

$$
\frac{k^{\prime}(\varphi)}{k(\varphi)+\epsilon}=\frac{k^{\prime}\left(\phi_{0}\right)}{k\left(\phi_{0}\right)}+\epsilon\left(\frac{k^{\prime \prime}\left(\phi_{0}\right) \phi_{1}}{k\left(\phi_{0}\right)}-\frac{k^{\prime}\left(\phi_{0}\right)\left(k^{\prime}\left(\phi_{0}\right) \phi_{1}+1\right)}{\left(k\left(\phi_{0}\right)\right)^{2}}\right)+\epsilon^{2}(\ldots) .
$$

Substituting this expansion into the diffusion equation and collecting the terms of order $\epsilon^{0}$, we obtain

$$
\partial_{\xi}^{2} u_{0}+\partial_{\xi}\left(\left(\rho-u_{0}\right) \frac{k^{\prime}\left(\phi_{0}\right)}{k\left(\phi_{0}\right)} \partial_{\xi} \phi_{0}\right)=0 \quad \text { for } \xi \leqslant \bar{\xi},
$$

with boundary conditions (see 211)

$$
\lim _{\xi \rightarrow \pm \infty} u_{0}(t, \xi, s)=u_{ \pm}(t, s),
$$

where $u_{ \pm}(t, s):=U_{0}\left(t, y_{0 \pm}(t, s)\right)$ denote the different limit values of the solutions to the sharp interface model at the moving interface from the different sides of the interface. We integrate (34) from $-\infty$ to $\bar{\xi}$ to arrive at

$$
\partial_{\xi} u_{0}=-\frac{\left(\rho-u_{0}\right) k^{\prime}\left(\phi_{0}\right)}{k\left(\phi_{0}\right)} \partial_{\xi} \phi_{0} \quad \text { at } \xi=\bar{\xi} .
$$

Note that this equation holds for all $\bar{\xi} \in \mathbb{R}$, and that the integration constant vanishes since $\partial_{\xi} u_{0}$ and $\partial_{\xi} \phi_{0}$ vanish as $\xi \rightarrow-\infty$ and $\left(k\left(\phi_{0}\right)\right)^{-1}$ remains bounded as $\phi_{0} \rightarrow-1$. Now we use the ansatz $u_{0}(t, \bar{\xi}, s)=\tilde{u}\left(t, \phi_{0}(\bar{\xi}), s\right)$, which gives for $\tilde{u}(t, y, s)$ the equation

$$
\partial_{y} \tilde{u}(t, y, s)=-\frac{(\rho-\tilde{u}(t, y, s)) k^{\prime}(y)}{k(y)} .
$$

This equation has the general solution

$$
\tilde{u}(t, y, s)=\rho+C(t, s) k(y),
$$

and we see that we cannot enforce a boundary condition for $y=1$ other than

$$
u_{0}(t,+\infty, s)=\tilde{u}(t, 1, s)=u_{+}(t, s)=\rho .
$$

If we use the boundary condition $\tilde{u}(t,-1, s)=u_{-}(t, s)$, the solution is given by $\tilde{u}(t, y, s)=$ $\rho+\left(u_{-}(t, s)-\rho\right) k(y)$, so that

$$
u_{0}(t, \xi, s)=\rho+\left(u_{-}(t, s)-\rho\right) k\left(\phi_{0}(\xi)\right) .
$$


REMARK If we supply initial conditions such that $U_{0}(0, x)=\rho$ where $\Phi_{0}(0, x)=1$, then the boundary conditions $u_{+}(t, s)=\rho$ at $\Gamma_{I}$ and (12) imply, together with the equation (31), that

$$
U_{0}(t, x)=\rho \quad \text { when } \Phi_{0}=1, \quad \text { and } \quad\left(\nabla U_{0}\left(t, y_{0+}(t, s)\right) \cdot n(t, s)\right)=0 .
$$

For the second problem, we collect the terms of order $\epsilon$ of the phase field equation and the diffusion equation. We arrive at the following equation for $\phi_{1}$ :

$$
-\partial_{\xi}^{2} \phi_{1}+p^{\prime \prime}\left(\phi_{0}\right) \phi_{1}=\left(\alpha v_{n, 0}+\kappa_{0}\right) \partial_{\xi} \phi_{0}-\beta k^{\prime}\left(\phi_{0}\right)\left(f\left(u_{0}\right)+\left(u_{0}-\rho\right) f^{\prime}\left(u_{0}\right)\right) .
$$

We can write this equation in the form

$$
L \phi_{1}=A\left(\phi_{0}\right)
$$

where

$$
\begin{aligned}
L \phi_{1} & =-\partial_{\xi}^{2} \phi_{1}+p^{\prime \prime}\left(\phi_{0}\right) \phi_{1} \\
A\left(\phi_{0}\right) & =\left(\alpha v_{n, 0}+\kappa_{0}\right) \partial_{\xi} \phi_{0}-\beta k^{\prime}\left(\phi_{0}\right)\left(f\left(u_{0}\right)+\left(u_{0}-\rho\right) f^{\prime}\left(u_{0}\right)\right) .
\end{aligned}
$$

The boundary conditions are in this case that $\phi_{1}( \pm \infty)$ is bounded (see (24)). Note that $L$ is a Fredholm operator of index zero. In particular, one has the following Fredholm alternative: the equation $L \phi_{1}=A\left(\phi_{0}\right)$ has a solution if and only if $A\left(\phi_{0}\right)$ is orthogonal to the finite-dimensional nullspace of $L^{*}$, i.e. of $L$. Since $\partial_{\xi} \phi_{0} \in \operatorname{ker}(L)$, the solvability condition implies

$$
\int_{-\infty}^{\infty} A\left(\phi_{0}\right) \partial_{\xi} \phi_{0} \mathrm{~d} \xi=0
$$

This can be written as

$$
\int_{-\infty}^{\infty}\left[\left(\alpha v_{n, 0}+\kappa_{0}\right)\left(\partial_{\xi} \phi_{0}\right)^{2}-\beta k^{\prime}\left(\phi_{0}\right) \partial_{\xi} \phi_{0}\left(f\left(u_{0}\right)+\left(u_{0}-\rho\right) f^{\prime}\left(u_{0}\right)\right)\right] \mathrm{d} \xi=0 .
$$

Substituting the solution 39 for $u_{0}$, we can rewrite this as

$$
\left(\alpha v_{n, 0}+\kappa_{0}\right) \int_{-\infty}^{\infty}\left(\partial_{\xi} \phi_{0}\right)^{2} \mathrm{~d} \xi=\beta \int_{-\infty}^{\infty} \partial_{\xi}\left[k\left(\phi_{0}\right) f\left(\rho+\left(u_{-}-\rho\right) k\left(\phi_{0}\right)\right)\right] \mathrm{d} \xi
$$

Performing the integration on the right hand side, we obtain the kinetic condition

$$
\alpha v_{n, 0}+\kappa_{0}=\frac{-\beta f\left(u_{-}\right)}{\int_{-\infty}^{\infty}\left(\partial_{\xi} \phi_{0}\right)^{2} \mathrm{~d} \xi}
$$

Since $\partial_{\xi} \phi_{0}=\left(\phi_{0}^{2}-1\right)^{2}$, we have $\int_{-\infty}^{\infty}\left(\partial_{\xi} \phi_{0}\right)^{2} \mathrm{~d} \xi=16 / 15$. If we choose

$$
\alpha=\sigma^{-1} \text { and } \beta=16 \alpha / 15 \text {, }
$$

we obtain the equivalent of equation (5):

$$
v_{n, 0}+\sigma \kappa_{0}=-f\left(u_{-}\right) .
$$


Now we collect the terms of order $\epsilon^{1}$ in the diffusion equation, which results in

$$
\begin{aligned}
-v_{n, 0} \partial_{\xi} u_{0}=D \partial_{\xi \xi} u_{1} & +\kappa_{0} D\left(\partial_{\xi} u_{0}+\left(\rho-u_{0}\right) h\left(\phi_{0}\right) \partial_{\xi} \phi_{0}\right) \\
+D \partial_{\xi} & {\left[\left(\rho-u_{0}\right)\left(h\left(\phi_{0}\right) \partial_{\xi} \phi_{1}+\left(\frac{k^{\prime \prime}\left(\phi_{0}\right) \phi_{1}}{k\left(\phi_{0}\right)}-\left(h\left(\phi_{0}\right)\right)^{2} \phi_{1}-\frac{h\left(\phi_{0}\right)}{k\left(\phi_{0}\right)}\right) \partial_{\xi} \phi_{0}\right)\right.} \\
& \left.-u_{1} h\left(\phi_{0}\right) \partial_{\xi} \phi_{0}\right],
\end{aligned}
$$

for $\xi \leqslant \bar{\xi}$, where we have used the notation

$$
h(\phi):=\frac{k^{\prime}(\phi)}{k(\phi)} .
$$

The boundary conditions follow from the matching conditions 27):

$$
\partial_{\xi} u_{1} \sim\left(\nabla u_{I} \cdot n\right)_{ \pm} \quad \text { for } \xi \rightarrow \pm \infty
$$

where

$$
\left(\nabla u_{I} \cdot n\right)_{ \pm}:=\nabla U_{0}\left(t, y_{0 \pm}(t, s)\right) \cdot n(t, s)
$$

denote the corresponding normal derivatives from the different sides of the interface. Using (35) and (39), equation 43, can be rewritten as

$$
\begin{aligned}
& -v_{n, 0} \partial_{\xi} u_{0}=D \partial_{\xi}\left(\partial_{\xi} u_{1}-u_{1} h\left(\phi_{0}\right) \partial_{\xi} \phi_{0}\right) \\
& \quad+D \partial_{\xi}\left[\left(\rho-u_{-}\right) k\left(\phi_{0}\right)\left(h\left(\phi_{0}\right) \partial_{\xi} \phi_{1}+\left(\frac{k^{\prime \prime}\left(\phi_{0}\right) \phi_{1}}{k\left(\phi_{0}\right)}-\left(h\left(\phi_{0}\right)\right)^{2} \phi_{1}-\frac{h\left(\phi_{0}\right)}{k\left(\phi_{0}\right)}\right) \partial_{\xi} \phi_{0}\right)\right] .
\end{aligned}
$$

Now we integrate with respect to $\xi$ to obtain

$$
\begin{aligned}
-v_{n, 0} u_{0}= & D\left(\partial_{\xi} u_{1}-u_{1} h\left(\phi_{0}\right) \partial_{\xi} \phi_{0}\right) \\
& +D\left[\left(\rho-u_{-}\right) k\left(\phi_{0}\right)\left(h\left(\phi_{0}\right) \partial_{\xi} \phi_{1}+\left(\frac{k^{\prime \prime}\left(\phi_{0}\right) \phi_{1}}{k\left(\phi_{0}\right)}-\left(h\left(\phi_{0}\right)\right)^{2} \phi_{1}-\frac{h\left(\phi_{0}\right)}{k\left(\phi_{0}\right)}\right) \partial_{\xi} \phi_{0}\right)\right]+C .
\end{aligned}
$$

The exact value of the integration constant $C$ will be determined later on. Some simple manipulations show that the equation above is equivalent to

$$
-v_{n, 0} u_{0}=D k\left(\phi_{0}\right) \partial \xi\left[\frac{u_{1}}{k\left(\phi_{0}\right)}+\left(\rho-u_{-}\right) \frac{k^{\prime}\left(\phi_{0}\right) \phi_{1}+1}{k\left(\phi_{0}\right)}\right]+C
$$

Next we want to take the limits $\bar{\xi} \rightarrow \pm \infty$. With 44, we write 47) as

$$
-v_{n, 0} u_{0}(\bar{\xi})=D\left(\partial_{\xi} b(\bar{\xi})-b(\bar{\xi}) h\left(\phi_{0}(\bar{\xi})\right) \partial_{\xi} \phi_{0}(\bar{\xi})\right)+C
$$

where

$$
b(\xi):=u_{1}(\xi)+\left(\rho-u_{-}\right)\left(k^{\prime}\left(\phi_{0}(\xi)\right) \phi_{1}(\xi)+1\right) .
$$


First we will consider the limit $\bar{\xi} \rightarrow-\infty$. Using $(45)$ and the fact that $\left(\nabla \Phi_{0}\left(t, y_{0-}(t, s)\right) \cdot n(t, s)\right)$ $=0$, the matching conditions $21,-28)$ give

$$
\left\{\begin{array}{l}
u_{1}(\xi) \sim \xi\left(\nabla u_{I} \cdot n\right)_{-}, \\
\partial_{\xi} u_{1}(\xi) \sim\left(\nabla u_{I} \cdot n\right)_{-}, \\
\phi_{0}(\xi) \sim-1, \\
\phi_{1}(\xi) \sim \Phi_{1}\left(t, y_{0-}\right), \quad \text { as } \xi \rightarrow-\infty \\
\partial_{\xi} \phi_{0}(\xi) \sim 0 \\
\partial_{\xi} \phi_{1}(\xi) \sim 0
\end{array}\right.
$$

These limits, together with the definitions (15) and (49) of $k$ and $b$, imply for $b(\xi)$ that

$$
\left\{\begin{array}{l}
b(\xi) \sim \xi\left(\nabla u_{I} \cdot n\right)_{-}+\rho-u_{-}, \quad \text { as } \xi \rightarrow-\infty . \\
\partial_{\xi} b(\xi) \sim\left(\nabla u_{I} \cdot n\right)_{-}
\end{array}\right.
$$

Substituting (14) into (33) results in $\partial_{\xi} \phi_{0}=\left(\phi_{0}^{2}-1\right)^{2}$. With separation of variables we obtain

$$
\xi=\frac{1}{4}\left(\ln \frac{1+\phi_{0}}{1-\phi_{0}}-\left(\phi_{0}-1\right)^{-1}-\left(\phi_{0}+1\right)^{-1}\right)+\tilde{C} .
$$

Now we see, using [44], (50, ) and $\phi_{0} \rightarrow-1$ as $\xi \rightarrow-\infty$, that

$$
\lim _{\xi \rightarrow-\infty} b(\xi) h\left(\phi_{0}(\xi)\right) \partial_{\xi} \phi_{0}(\xi)=0,
$$

since $h\left(\phi_{0}(\xi)\right)$ remains bounded for $\xi \rightarrow-\infty$. We use this in letting $\bar{\xi} \rightarrow-\infty$ in (48) and obtain

$$
-v_{n, 0} u_{-}=D\left(\nabla u_{I} \cdot n\right)_{-}+C .
$$

Secondly we consider the limit $\bar{\xi} \rightarrow+\infty$. From equation (33) for $\phi_{0}$ and the definitions of $p(\varphi), k(\varphi)$ and $h(\varphi)$ we find

$$
\begin{aligned}
\lim _{\xi \rightarrow \infty} h\left(\phi_{0}\right) \partial_{\xi} \phi_{0} & =\lim _{\xi \rightarrow \infty} \frac{k^{\prime}\left(\phi_{0}\right)}{k\left(\phi_{0}\right)} \partial_{\xi} \phi_{0}=\lim _{\xi \rightarrow \infty} 2 \frac{\left(\phi_{0}^{2}-1\right)^{2}}{\left(1-\phi_{0}\right)^{2}}\left(\phi_{0}-1\right) \\
& =\lim _{\xi \rightarrow \infty} 2\left(\phi_{0}+1\right)^{2}\left(\phi_{0}-1\right)=0
\end{aligned}
$$

since $\phi_{0}$ tends to 1 as $\xi \rightarrow \infty$.

Using the fact that $\left(\nabla U_{0}\left(t, y_{0+}(t, s) \cdot n(t, s)\right)=\left(\nabla \Phi_{0}\left(t, y_{0+}(t, s)\right) \cdot n(t, s)\right)=0\right.$, the matching conditions (21)-28) give

$$
\left\{\begin{array}{l}
u_{1}(\xi) \sim U_{1}\left(t, y_{0+}(t, s)\right), \\
\partial_{\xi} u_{1}(\xi) \sim 0, \\
\phi_{0}(\xi) \sim 1, \\
\phi_{1}(\xi) \sim \Phi_{1}\left(t, y_{0+}(t, s)\right), \quad \text { as } \xi \rightarrow+\infty . \\
\partial_{\xi} \phi_{0}(\xi) \sim 0, \\
\partial_{\xi} \phi_{1}(\xi) \sim 0
\end{array}\right.
$$


These limits imply, again together with the definitions (15) and 49) of $k$ and $b$, that

$$
\left\{\begin{array}{l}
b(\xi) \sim U_{1}\left(t, y_{0+}(t, s)\right)+\left(\rho-u_{-}\right), \\
\partial_{\xi} b(\xi) \sim 0
\end{array}\right.
$$

Now we send $\bar{\xi}$ to $+\infty$ in $(48)$ and, using $(52)$ and $(53)$, we obtain

$$
-v_{n, 0} \rho=C
$$

Subtracting (54) from (51) results in the Rankine-Hugoniot condition

$$
v_{n, 0}\left(\rho-u_{-}\right)=D\left(\nabla u_{I} \cdot n\right)_{-} .
$$

We now collect the equations 29]-(30), 38, 40, 41) (together with the choice for $\alpha$ and $\beta$ in (42) ) and (55), which we obtained for the first order terms $U_{0}$ and $\Phi_{0}$. We define the domains $\Omega^{+}(t):=\left\{x \in \Omega \mid \Phi_{0}(t, x)=1\right\}$ and $\Omega^{-}(t):=\left\{x \in \Omega \mid \Phi_{0}(t, x)=-1\right\}$, with common boundary $\Gamma_{I}(t)$, and write

$$
\begin{aligned}
\partial_{t} U_{0}=D \Delta U_{0} & \text { on } \Omega^{-}(t), \\
U_{0}=\rho & \text { on } \Omega^{+}(t), \\
v_{n, 0}\left(\rho-U_{0}^{-}\right)=D\left(\nabla U_{0} \cdot n\right)^{-} & \text {on } \Gamma_{I}(t), \\
v_{n, 0}+\sigma \kappa_{0}=-f\left(U_{0}^{-}\right) & \text {on } \Gamma_{I}(t),
\end{aligned}
$$

where we denote by the "-"-superscripts the limit values at $\Gamma_{I}(t)$ from the side where $\Phi_{0}=-1$. Supplementing the equations above with appropriate initial and boundary conditions, we see that we have now obtained the sharp interface equations (4)-(5).

REMARKS 1. The reason for choosing the particular double well potential given in (14) is the limit $\lim _{\xi \rightarrow \infty} h\left(\phi_{0}\right) \partial_{\xi} \phi_{0}$, which should be equal to zero, as we have seen above (see (52)), to obtain the Rankine-Hugoniot condition in the sharp interface limit. With the classical choice of the double well potential as given in (13), $\lim _{\xi \rightarrow \infty} h\left(\phi_{0}\right) \partial_{\xi} \phi_{0}$ is not equal to zero.

As mentioned in Section 3 , there is some freedom in choosing the functions $p$ and $k$. In this section we have seen that it is their behaviour near $\varphi= \pm 1$ that is important for the asymptotic analysis. This means that for different functions, but with the same behaviour near \pm 1 , the same formal asymptotic results can be obtained.

2. The asymptotic analysis as presented above does, with slight variations, also apply if we use the equation

$$
\partial_{t} u-D \nabla \cdot\left(\nabla u+(\rho-u) \frac{k^{\prime}(\varphi)}{k(\varphi)} \nabla \varphi\right)=0
$$

instead of equation (8). In fact, (8) is a regularized version of the equation above, where the coefficient $(\rho-u) k^{\prime}(\varphi) / k(\varphi)$ has a singularity for $\varphi=1$. In Section 5 we prove existence and uniqueness of solutions to the regularized phase field model, and we also prove the existence of solutions to the singular version when the initial condition $\varphi_{0}$ satisfies the condition $\ln \left(1-\varphi_{0}\right) \in$ $H^{1}(\Omega)$. 


\section{Solvability of the phase field model}

The phase field model that will be analyzed in this section has the form

$$
\begin{aligned}
& \alpha \epsilon^{2} \partial_{t} \varphi-\epsilon^{2} \Delta \varphi+p^{\prime}(\varphi)+\epsilon \beta q(u, \varphi)=0, \\
& \partial_{t} u-D \Delta u-D \nabla \cdot(g(u, \varphi) \nabla \varphi)=0,
\end{aligned}
$$

with some functions $q$ and $g$. The original form of $q$ and $g$ is

$$
\begin{aligned}
& q(u, \varphi)=k^{\prime}(\varphi)\left(f(u)-(\rho-u) f^{\prime}(u)\right), \\
& g(u, \varphi)=\frac{(\rho-u) k^{\prime}(\varphi)}{k(\varphi)} .
\end{aligned}
$$

The main difficulty here is the singularity of the function $g$ that is generated by the root $k(1)=0$. We will assume that

$$
\bar{g}(u, \varphi)=g(u, \varphi)(1-\varphi)
$$

is a uniformly bounded Lipschitz function. In fact this contradicts the form (59) of $g$, because the factor $\rho-u$ is not bounded. However, since only the values $u \in(0, \rho)$ are physically realistic, we may replace this factor by a bounded term, for example by

$$
\rho-u \mapsto \begin{cases}0, & u>\rho, \\ \rho-u, & 0 \leqslant u \leqslant \rho \\ \rho, & u<0 .\end{cases}
$$

For the double well potential we assume

$$
\begin{aligned}
p(\varphi) & \geqslant c_{0}|\varphi|^{\gamma}-c_{1}, \\
\left|p^{\prime}(\varphi)-p^{\prime}(\psi)\right| & \leqslant c_{2}\left(|\varphi|^{\gamma-2}+|\psi|^{\gamma-2}+1\right)|\varphi-\psi|
\end{aligned}
$$

with $c_{0}, c_{1}, c_{2}>0$ and some $2 \leqslant \gamma<+\infty$. For $q$ we assume

$$
\begin{aligned}
|q(u, \varphi)| & \leqslant c_{1}\left(|\varphi|^{\gamma / 2}+|u|+1\right), \\
|q(u, \varphi)-q(v, \psi)| & \leqslant c_{2}\left(|\varphi|^{\gamma / 2}+|\psi|^{\gamma / 2}+1\right)(|u-v|+|\varphi-\psi|)
\end{aligned}
$$

with the same $\gamma$ as above. Further conditions for $g$ are specified below.

The system of equations (56)-57) with initial conditions and boundary conditions given by (9)-(12) has the following weak formulation: Find functions $u, \varphi \in L_{2}\left(J ; H^{1}(\Omega)\right)$ with $\partial_{t} u, \partial_{t} \varphi \in$ $L_{2}\left(J ; H^{1}(\Omega)^{*}\right)$ that satisfy the initial conditions $(9-10)$ such that for all test functions $v, w \in$ $L_{2}\left(J ; H^{1}(\Omega)\right)$,

$$
\begin{aligned}
& \left\langle\partial_{t} u, v\right\rangle+\int_{Q} D(\nabla u+g(u, \varphi) \nabla \varphi) \cdot \nabla v \mathrm{~d} x \mathrm{~d} t=0, \\
& \left\langle\alpha \epsilon^{2} \partial_{t} \varphi, w\right\rangle+\int_{Q}\left(\epsilon^{2} \nabla \varphi \cdot \nabla w+\left(p^{\prime}(\varphi)+\epsilon \beta q(u, \varphi)\right) w\right) \mathrm{d} x \mathrm{~d} t=0 .
\end{aligned}
$$

Here $Q=J \times \Omega$ denotes the time-space cylinder. 


\subsection{Analysis for a regularized problem}

We first consider the phase field model (64), (65) under the assumption that the function $g$ has no singularity. We assume that $p, q$ satisfy the conditions (62), 63) and 69 below and that $g$ is bounded and globally Lipschitz.

The analysis is done by a standard Galerkin approximation. Let $\left\{\psi_{m}\right\}_{m \in \mathbb{N}}$ be the sequence of eigenfunctions to the problem of finding $\psi \in H^{1}(\Omega)$ such that

$$
\int_{\Omega} \nabla \psi \cdot \nabla v \mathrm{~d} x=\lambda \int_{\Omega} \psi v \mathrm{~d} x \quad \text { for every } v \in H^{1}(\Omega),
$$

corresponding to the nondecreasing sequence of eigenvalues $\left\{\lambda_{m}\right\}_{m \in \mathbb{N}}$, and let $V_{m}=$ $\operatorname{span}\left\{\psi_{1}, \ldots, \psi_{m}\right\}$. Then $\bigcup_{m \in \mathbb{N}} V_{m}$ is dense in $H^{1}(\Omega)$ and the orthogonal projection $\pi_{m}$ of $H^{1}(\Omega)$ onto $V_{m}$ with respect to the scalar product of $L_{2}(\Omega)$ is bounded with respect to the norm of $H^{1}(\Omega)$ uniformly in $m$. These properties that follow from the special choice of the basis will be mathematically convenient to derive a "dual estimate" below. They are, however, not strictly required for the existence proof. For a sufficiently regular domain (for example a $C^{2}$ domain) we have

$$
V_{m} \subset L_{\infty}(\Omega)
$$

by elliptic regularity theory. The Galerkin approximation of level $m$ to 64, 65) is a pair $\left(u_{m}, \varphi_{m}\right) \in$ $C^{1}\left(J ; V_{m}\right)$ of functions that satisfy the approximate initial conditions

$$
u_{m}(0, \cdot)=\pi_{m} u_{0} \quad \text { and } \quad \varphi_{m}(0, \cdot)=\pi_{m} \varphi_{0},
$$

and the variational equations (64), 65) for test functions $v, w \in L_{2}\left(J ; V_{m}\right)$. The Galerkin equations can be formulated as a system of ordinary differential equations for the coefficients in the representation of $u_{m}, \varphi_{m}$ with respect to the basis of $V_{m}$. The existence and uniqueness of a solution follows from the standard theory of ordinary differential equations.

In order to pass to the limit $m \rightarrow+\infty$, we need a priori estimates that are independent of $m$. The first estimate is derived by using the test functions $v=\chi_{t_{0}} u_{m}$ and $w=\chi_{t_{0}} \partial_{t} \varphi_{m}$ with the indicator function $\chi_{t_{0}}$ of the time interval $\left(0, t_{0}\right)$ in $\left.(64), 65\right)$. With the help of the representations and estimates

$$
\begin{aligned}
p^{\prime}\left(\varphi_{m}\right) \partial_{t} \varphi_{m} & =\partial_{t}\left(p\left(\varphi_{m}\right)\right), \\
\left|q\left(u_{m}, \varphi_{m}\right) \partial_{t} \varphi_{m}\right| & \leqslant c\left(\left|\partial_{t} \varphi_{m}\right|^{2}+\left|\varphi_{m}\right|^{\gamma}+\left|u_{m}\right|^{2}+1\right)
\end{aligned}
$$

and the application of suitable Hölder inequalities and the Gronwall Lemma we derive

$$
\begin{aligned}
\left\|\partial_{t} \varphi_{m}\right\|_{L_{2}(Q)}^{2}+\left\|\nabla \varphi_{m}\right\|_{L_{\infty}\left(J ; L_{2}(\Omega)\right)}^{2}+\left\|p\left(\varphi_{m}\right)\right\|_{L_{\infty}\left(J ; L_{1}(\Omega)\right)} & \\
& +\left\|u_{m}\right\|_{L_{\infty}\left(J ; L_{2}(\Omega)\right)}^{2}+\left\|\nabla u_{m}\right\|_{L_{2}(Q)}^{2} \leqslant c
\end{aligned}
$$

with some constant $c$ that depends on the initial conditions and the coefficients and coefficient functions of (64), 65), but not on $m$. In order to obtain some time regularity of $u_{m}$ we consider the following dual estimate: for $v \in L_{2}\left(J ; H^{1}(\Omega)\right)$ we use $\pi_{m} v \in L_{2}\left(J ; V_{m}\right)$ as test function in 64). Due to $\left\langle\partial_{t} u_{m}, \pi_{m} v\right\rangle=\left\langle\partial_{t} u_{m}, v\right\rangle$ and $\left\|\pi_{m} v\right\|_{H^{1}(\Omega)} \leqslant c\|v\|_{H^{1}(\Omega)}$ with $c$ independent of $m$ we obtain

$$
\left\|\partial_{t} u_{m}\right\|_{L_{2}\left(J ; H^{1}(\Omega)^{*}\right)} \leqslant c
$$


with $c$ independent of $m$. Interpolation with $u_{m} \in L_{2}\left(J ; H^{1}(\Omega)\right)$ yields

$$
\left\|u_{m}\right\|_{H^{1 / 2}\left(J ; L_{2}(\Omega)\right)} \leqslant c
$$

with $c$ independent of $m$.

As a consequence of these a priori estimates, there is a sequence $m_{k} \rightarrow+\infty$ of Galerkin parameters, a sequence $\left(u_{k}, \varphi_{k}\right)=\left(u_{m_{k}}, \varphi_{m_{k}}\right)$ of solutions and a limit $(u, \varphi) \in L_{2}\left(J ; H^{1}(\Omega)\right)^{2} \cap$ $H^{1}\left(J ; H^{1}(\Omega)^{*}\right)$ such that $u_{k} \rightarrow u, \varphi_{k} \rightarrow \varphi$ weakly in $L_{2}\left(J ; H^{1}(\Omega)\right)$ and $\partial_{t} u_{k} \rightarrow \partial_{t} u, \partial_{t} \varphi_{k} \rightarrow \partial_{t} \varphi$ weakly in $L_{2}\left(J ; H^{1}(\Omega)^{*}\right)$. Let $H^{1 / 2,1}(Q)$ be the anisotropic Sobolev space with time regularity $1 / 2$ and space regularity 1 , that is, $H^{1 / 2,1}(Q)=H^{1 / 2}\left(J ; L_{2}(\Omega)\right) \cap L_{2}\left(J ; H^{1}(\Omega)\right)$. By the embedding $H^{1 / 2,1}(Q) \hookrightarrow H^{\lambda / 2}\left(J ; H^{1-\lambda}(\Omega)\right)$ for arbitrary $\lambda \in(0,1)$ (see e.g. Theorem 6.4.5 in [1]) and the usual compact embeddings of Sobolev spaces we conclude the compact embedding $H^{1 / 2,1}(Q) \hookrightarrow$ $L_{r}(Q)$ with $r$ such that $\lambda / 2-1 / 2>-1 / r$ and $(1-\lambda)-d / 2>-d / r$. Choosing $\lambda$ in an optimal way we conclude $r<2+4 / d$. Altogether we have the compact embedding

$$
H^{1 / 2,1}(Q) \hookrightarrow L_{r}(Q) \quad \text { for any } r<2+4 / d .
$$

Hence, for any such $r$ we can extract a subsequence such that $u_{k} \rightarrow u$ and $\varphi_{k} \rightarrow \varphi$ almost everywhere in $Q$ and strongly in $L_{r}(Q)$. Relations 67 and 62 imply an upper bound for $\left\|\varphi_{k}\right\|_{L_{\infty}\left(J ; L_{\gamma}(\Omega)\right)}$. From 62 ) we also find $\left|p^{\prime}\left(\varphi_{k}\right)\right| \leqslant c_{3}+c_{4}\left|\varphi_{k}\right|^{\gamma-1}$. Together with 63) this implies that $p^{\prime}\left(\varphi_{k}\right)$ and $q\left(u_{k}, \varphi_{k}\right)$ are bounded in $L_{2}\left(J ; L_{\gamma /(\gamma-1)}(\Omega)\right)$. Hence we can choose our subsequence such that $\varphi_{k} \rightarrow \varphi$ weakly in $L_{\gamma}(Q)$ and, by interpolation with the strong convergence in $L_{r}(Q)$, strongly in $L_{\gamma / 2}(Q), p^{\prime}\left(\varphi_{k}\right) \rightarrow \zeta$ and $q\left(u_{k}, \varphi_{k}\right) \rightarrow \xi$ in $L_{2}\left(J ; L_{\gamma /(\gamma-1)}(\Omega)\right)$ with suitable limits $\zeta, \xi$. Moreover, from the continuity estimates of (62) and 63) we have $p^{\prime}\left(\varphi_{k}\right) \rightarrow$ $p^{\prime}(\varphi)$ and $q\left(u_{k}, \varphi_{k}\right) \rightarrow q(u, \varphi)$ in $L_{1}(Q)$. Since both the weak limits in $L_{2}\left(J ; L_{\gamma /(\gamma-1)}(\Omega)\right)$ and the strong limits in $L_{1}(Q)$ must be the same we have $p^{\prime}\left(\varphi_{k}\right) \rightarrow p^{\prime}(\varphi)$ and $q\left(u_{k}, \varphi_{k}\right) \rightarrow q(u, \varphi)$ in $L_{2}\left(J ; L_{\gamma /(\gamma-1)}(\Omega)\right)$.

We now pass to the limit $k \rightarrow+\infty$ in the Galerkin equations of level $m_{k}$ with solution $\left(u_{k}, \varphi_{k}\right)$ and test functions $v, w \in L_{2}\left(J ; V_{m}\right)$ with fixed $m$. Since $g$ is globally bounded and Lipschitz and $u_{k} \rightarrow u, \varphi_{k} \rightarrow \varphi$ a.e., we find by the Lebesgue dominated convergence theorem that

$$
\int_{Q}\left|g\left(u_{k}, \varphi_{k}\right) \nabla v-g(u, \varphi) \nabla v\right|^{2} \mathrm{~d} x \mathrm{~d} t \rightarrow 0
$$

as $k \rightarrow+\infty$. This implies

$$
\int_{Q} g\left(u_{k}, \varphi_{k}\right) \nabla \varphi_{k} \cdot \nabla v \mathrm{~d} x \mathrm{~d} t \rightarrow \int_{Q} g(u, \varphi) \nabla \varphi \cdot \nabla v \mathrm{~d} x \mathrm{~d} t
$$

as $k \rightarrow+\infty$. From the weak convergences $p^{\prime}\left(\varphi_{k}\right) \rightarrow p^{\prime}(\varphi)$ and $q\left(u_{k}, \varphi_{k}\right) \rightarrow q(u, \varphi)$ in $L_{2}\left(J ; L_{\gamma /(\gamma-1)}(\Omega)\right)$ and the property $w \in L_{2}\left(J ; V_{m}\right) \subset L_{2}\left(J ; L_{\infty}(\Omega)\right)$ (see 66$)$, we find

$$
\int_{Q}\left(p^{\prime}\left(\varphi_{k}\right)+\epsilon \beta q\left(u_{k}, \varphi_{k}\right)\right) w \mathrm{~d} x \mathrm{~d} t \rightarrow \int_{Q}\left(p^{\prime}(\varphi)+\epsilon \beta q(u, \varphi)\right) w \mathrm{~d} x \mathrm{~d} t .
$$

The convergence of the other terms in the weak formulations follows easily. Hence $(\varphi, u)$ solves 64), 65 for arbitrary test functions $v, w \in L_{2}\left(J ; V_{m}\right)$ with arbitrary $m \in \mathbb{N}$. Since $\bigcup_{m \in \mathbb{N}} V_{m}$ is dense in $H^{1}(\Omega),(u, \varphi)$ is a solution of (64), 65] for any test functions $v, w \in L_{2}\left(J ; H^{1}(\Omega)\right)$. 
In order to prove uniqueness of the weak solution, we first need some additional regularity of the phase field $\varphi$. Using the weak maximum principle and the additional requirements $\left|\varphi_{0}\right| \leqslant 1$ for the initial conditions and

$$
\begin{array}{ll}
p^{\prime}(\varphi)+\epsilon \beta q(u, \varphi) \geqslant 0 & \text { for } \varphi \geqslant 1, \\
p^{\prime}(\varphi)+\epsilon \beta q(u, \varphi) \leqslant 0 & \text { for } \varphi \leqslant-1,
\end{array}
$$

we obtain $|\varphi| \leqslant 1$ almost everywhere. By the application of standard regularity results for linear parabolic equations (see e.g. [12, Theorem 5.4]) to the phase field equation with $p^{\prime}(\varphi)+\epsilon \beta q(u, \varphi)$ interpreted as a given right hand side we prove $\varphi \in W_{s}^{1,2}(Q)$ for an arbitrary $s \leqslant 2+4 / d$, provided $\Omega$ is sufficiently regular and the initial condition satisfies $\varphi_{0} \in W_{s}^{2-2 / s}(\Omega)$. Here $W_{s}^{1,2}(Q)=$ $W_{s}^{1}\left(J ; L_{s}(Q)\right) \cap L_{s}\left(J ; W_{s}^{2}(Q)\right)$; and the condition $s \leqslant 2+4 / d$ is necessary to ensure $p^{\prime}(\varphi)+$ $\epsilon \beta q(u, \varphi) \in L_{s}(Q)$ via the embedding $H^{1 / 2,1}(Q) \hookrightarrow L_{s}(Q)$.

We now consider two possibly different solutions $\left(u^{(j)}, \varphi^{(j)}\right), j=1$, 2. From the regularity just obtained and the embedding $W_{s}^{1,2}(Q) \hookrightarrow W_{s}^{\lambda}\left(J ; W_{s}^{2(1-\lambda)}(\Omega)\right) \hookrightarrow L_{\infty}\left(J ; W_{r}^{1}(\Omega)\right)$ with $\lambda>1 / s$ and $2(1-\lambda)-d / s>1-d / r$ we conclude $\varphi^{(j)} \in L_{\infty}\left(J ; W_{r}^{1}(\Omega)\right), j=1,2$, provided $1 / r>$ $1 / s+2 / d s-1 / d$. We use the test functions $v=\chi_{t}\left(u^{(1)}-u^{(2)}\right)$ and $w=\mu \chi_{t}\left(\varphi^{(1)}-\varphi^{(2)}\right)$ with some sufficiently large $\mu$ in the difference of the equations 64, 65] for the two different solutions and exploit the Lipschitz properties of $p, q$ and $g$ as well as standard Hölder estimates. In particular we use the estimates

$$
\begin{aligned}
& \left|\left(g\left(u^{(1)}, \varphi^{(1)}\right) \nabla \varphi^{(1)}-g\left(u^{(2)}, \varphi^{(2)}\right) \nabla \varphi^{(2)}\right) \cdot \nabla\left(u^{(1)}-u^{(2)}\right)\right| \\
& \quad \leqslant c_{1}\left|\nabla \varphi^{(1)}\right|^{2}\left(\left|u^{(1)}-u^{(2)}\right|^{2}+\left|\varphi^{(1)}-\varphi^{(2)}\right|^{2}\right)+c_{2}\left|\nabla\left(\varphi^{(1)}-\varphi^{(2)}\right)\right|^{2}+\frac{D}{2}\left|\nabla\left(u^{(1)}-u^{(2)}\right)\right|^{2}
\end{aligned}
$$

and

$$
\begin{aligned}
& \int_{0}^{t} \int_{\Omega}\left|\nabla \varphi^{(1)}\right|^{2}\left(\left|u^{(1)}-u^{(2)}\right|^{2}+\left|\varphi^{(1)}-\varphi^{(2)}\right|^{2}\right) \mathrm{d} x \mathrm{~d} t \\
& \quad \leqslant\left\|\nabla \varphi^{(1)}\right\|_{L_{\infty}\left((0, t) ; L_{r}(\Omega)\right)}^{2}\left(\left\|u^{(1)}-u^{(2)}\right\|_{L_{2}\left((0, t) ; L_{\sigma}(\Omega)\right)}^{2}+\left\|\varphi^{(1)}-\varphi^{(2)}\right\|_{L_{2}\left((0, t) ; L_{\sigma}(\Omega)\right)}^{2}\right)
\end{aligned}
$$

with $2 / r+2 / \sigma=1$. The contribution of $u^{(1)}-u^{(2)}$ can be further estimated by

$$
\left\|u^{(1)}-u^{(2)}\right\|_{L_{2}\left((0, t) ; L_{\sigma}(\Omega)\right)}^{2} \leqslant \eta\left\|u^{(1)}-u^{(2)}\right\|_{L_{2}\left((0, t) ; H^{1}(\Omega)\right)}^{2}+c(\eta)\left\|u^{(1)}-u^{(2)}\right\|_{L_{2}((0, t) \times \Omega)}^{2}
$$

with arbitrarily small $\eta>0$, if $1-d / 2>-d / \sigma$. Combining the conditions for $r$ and $\sigma$ we find

$$
\frac{1}{2}-\frac{1}{d}<\frac{1}{\sigma}<\frac{1}{2}-\frac{1}{s}-\frac{2}{d s}+\frac{1}{d} .
$$

Hence we can choose any value $s>1+d / 2$ if $d \leqslant 3$. Then we find the estimate

$$
\begin{aligned}
\left\|u^{(1)}(t)-u^{(2)}(t)\right\|_{L_{2}(\Omega)}^{2}+ & \left\|\varphi^{(1)}(t)-\varphi^{(2)}(t)\right\|_{L_{2}(\Omega)}^{2} \\
& +\left\|\nabla\left(u^{(1)}-u^{(2)}\right)\right\|_{L_{2}((0, t) \times \Omega)}^{2}+\left\|\nabla\left(\varphi^{(1)}-\varphi^{(2)}\right)\right\|_{L_{2}((0, t) \times \Omega)}^{2} \\
\leqslant & c_{1}\left(\left\|u^{(1)}-u^{(2)}\right\|_{L_{2}((0, t) \times \Omega)}^{2}+\left\|\varphi^{(1)}(t)-\varphi^{(2)}(t)\right\|_{L_{2}((0, t) \times \Omega)}^{2}\right) .
\end{aligned}
$$

Application of the Gronwall Lemma shows the uniqueness. This concludes the proof of the following theorem: 
THEOREM 1 Let $\alpha, \epsilon, D$ be positive parameters. Let $\Omega$ be a bounded $C^{2}$ domain of dimension $d \in\{2,3\}$, let $p: \mathbb{R} \rightarrow \mathbb{R}$ and $q: \mathbb{R}^{2} \rightarrow \mathbb{R}$ satisfy the conditions 62, 63, and 69, let $g$ be bounded and globally Lipschitz, and let $u_{0} \in L_{2}(\Omega), \varphi_{0} \in W_{s}^{2-2 / s}(\Omega)$ for $s>1+d / 2$ and $\left|\varphi_{0}\right| \leqslant 1$. Then the weak formulation (64), 65) has a unique solution $(u, \varphi)$ with $\varphi \in W_{s}^{1,2}(Q)$ and $|\varphi| \leqslant 1$ almost everywhere.

REMARKS 1. The Lipschitz property of $p, q$ and $g$ is required for the proof of the uniqueness result; for the existence proof it is sufficient to have continuity and appropriate growth conditions.

2. The conditions on the smoothness of the domain here are formulated to ensure the regularity properties for the Galerkin basis and the phase field to be used. Some additional regularity of the phase field is necessary for the proof of uniqueness, due to the coefficient function $g(u, \varphi)$ that depends on the solution. The regularity of the basis $V_{m}$ is just required for simplicity of the presentation, it can be cirumvented by some additional technicalities.

3 . It is easily checked that both the double well potentials (13) and (14) satisfy the conditions in (62). The condition (63) is satisfied for $q(u, \varphi)$ as in (58) with the choice of $k(\varphi)$ as in (15) and for $f(u)$ that has bounded first derivatives, since for this choice $k^{\prime}(\varphi)$ is bounded. The condition 632) holds for $q(u, \varphi)$ as in (58) and $k(\varphi)$ as in (15) if $f(u)-(\rho-u) f^{\prime}(u)$ is bounded and $f^{\prime}$ is bounded and Lipschitz. If this is not the case for a particular choice of $f(u)$, then one may need to replace this term with a bounded version by cutting the term for unphysical values of $u$ as in (61). The conditions in (69) are satisfied for both (13) and (14) together with (15) and $q$ given by (58), since for the $k(\varphi)$ in $\left[15\right.$ we have $k^{\prime}(\varphi)=0$ for $\varphi \leqslant-1$ and $\varphi \geqslant 1$.

\subsection{Analysis of the singular case}

The analysis for the singular case will be done by the following approach: we first regularize the term $g(u, \varphi)$ by

$$
g_{\delta}(u, \varphi)=\frac{\bar{g}(u, \varphi)}{[1-\varphi]_{+}+\delta}
$$

with the $\bar{g}$ from (60), where $[x]_{+}=\max \{x, 0\}$ is the positive part of $x$. In addition to 62, 63) and (69) we also assume

$$
p^{\prime}(\varphi)=(1-\varphi) \bar{p}(\varphi), \quad q(u, \varphi)=(1-\varphi) \bar{q}(u, \varphi)
$$

with Lipschitz functions $\bar{p}, \bar{q}$ that are uniformly bounded for $\varphi \in[-1,1]$ and $u \in \mathbb{R}$.

The existence and uniqueness of solutions to the modified problem as well as the property $|\varphi| \leqslant 1$ a.e. follow from Theorem 1 . Then we plan to pass to the limit $\delta \rightarrow 0$. For this it is necessary to have a priori estimates that are independent of $\delta$. Using the test function $w=\chi_{t_{0}} \partial_{t} \varphi$, where $\chi_{t_{0}}$ is the indicator function of the time interval $\left(0, t_{0}\right)$, in 65 and applying the Gronwall Lemma yields

$$
\left\|\partial_{t} \varphi\right\|_{L_{2}(Q)}+\|\nabla \varphi\|_{L_{\infty}\left(J ; H^{1}(\Omega)\right)} \leqslant c
$$

with $c$ independent of $\delta$.

For the following considerations we make the assumptions $\varphi_{0}<1$ and

$$
\ln \left(1-\varphi_{0}\right) \in H^{1}(\Omega)
$$

for the initial data. This seems to be a little strange at a first view, because $\varphi=1$ corresponds to the solid phase. However, from the asymptotic analysis done in the previous section we see that the 
first order term $\phi_{0}(\xi)$ in the asymptotic expansion is the solution to the initial value problem (33). Due to $p( \pm 1)=0$ this solution approaches the values \pm 1 as $\xi \rightarrow \pm \infty$, but it does not attain these values: if there is a point $\xi_{0}$ with $\phi_{0}\left(\xi_{0}\right)=1$, then $\phi_{0}$ is also a solution to the problem

$$
\phi^{\prime}(\xi)=\sqrt{2 p(\phi(\xi))} \quad \text { for } \xi \in \mathbb{R}, \quad \phi\left(\xi_{0}\right)=1 ;
$$

and the unique solution to this problem is $\phi_{0}(\xi)=1$. The variable $\xi$ here stands for $\xi=r / \epsilon$, where $r=r(x)$ is a signed distance to the sharp interface. If $\epsilon$ is fixed, then it makes sense to use initial data that satisfy [71), for example by choosing $\varphi_{0}(x)=\phi_{0}(r(x) / \epsilon)$. For fixed $\epsilon$ the argument $|r(x)| / \epsilon \leqslant \operatorname{diam}(\Omega) / \epsilon$ is bounded, hence we find $\phi_{0}(r(x) / \epsilon) \leqslant \Phi_{0}(\operatorname{diam}(\Omega) / \epsilon)<1$ and therefore $\left|\ln \left(1-\phi_{0}\right)\right| \leqslant c$ with some $c<+\infty$.

We now use the test function $w=\chi_{t_{0}}(1-\varphi+\delta)^{-1}$, where $\chi_{t_{0}}$ denotes again the indicator function of the time interval $\left(0, t_{0}\right)$, in (65). This function is well defined because $\varphi \leqslant 1$ and $\delta>0$. We use the relations $\partial_{t} \varphi w=-\partial_{t} \ln (1-\varphi+\delta), \nabla \varphi \cdot \nabla w=(1-\varphi+\delta)^{-2}|\nabla \varphi|^{2}=|\nabla \ln (1-\varphi+\delta)|^{2}$ for $t \in\left(0, t_{0}\right)$ and the estimates

$$
\left|p^{\prime}(\varphi)(1-\varphi+\delta)^{-1}\right| \leqslant|\bar{p}(\varphi)| \leqslant c_{1}, \quad\left|q(u, \varphi)(1-\varphi+\delta)^{-1}\right| \leqslant|\bar{q}(u, \varphi)| \leqslant c_{2},
$$

which are a consequence of $(70)$ and the property $\varphi \in[-1,1]$, and obtain

$$
\alpha \epsilon^{2} \int_{\Omega}\left(\ln \left(1-\varphi_{0}+\delta\right)-\ln \left(1-\varphi\left(t_{0}\right)+\delta\right)\right) \mathrm{d} x+\epsilon^{2} \int_{0}^{t_{0}} \int_{\Omega}|\nabla \ln (1-\varphi+\delta)|^{2} \mathrm{~d} x \mathrm{~d} t \leqslant c
$$

with some $c$ independent of $\delta$. From $\varphi \in[-1,1]$ and the properties $-\ln (1-\varphi+\delta)=|\ln (1-\varphi+\delta)|$ for $\varphi \geqslant \delta$ and $|\ln (1-\varphi+\delta)| \leqslant \ln (3)$ for $\varphi \leqslant \delta$ and $\delta<1$ we conclude

$$
\int_{\Omega}\left(-\ln \left(1-\varphi\left(t_{0}\right)+\delta\right)\right) \mathrm{d} x \geqslant\left\|\ln \left(1-\varphi\left(t_{0}\right)+\delta\right)\right\|_{L_{1}(\Omega)}-c
$$

with some $c$ independent of $\delta$ for $\delta \in(0,1)$. This shows

$$
\|\ln (1-\varphi+\delta)\|_{L_{\infty}\left(J ; L_{1}(\Omega)\right)}+\|\nabla \ln (1-\varphi+\delta)\|_{L_{2}(Q)}^{2} \leqslant c
$$

with $c$ independent of $\delta$. By the Poincaré inequality

$$
\|v\|_{L_{2}(\Omega)} \leqslant c_{1}\|\nabla v\|_{L_{2}(\Omega)}+c_{2}\|v\|_{L_{1}(\Omega)}
$$

applied to $v=\ln (1-\varphi+\delta)$, we then derive

$$
\|\ln (1-\varphi+\delta)\|_{L_{2}(Q)} \leqslant c .
$$

Then we take the test function $v=u$ in 64). From (72) and the uniform bound of $\bar{g}$ we find

$$
\left\|g_{\delta}(u, \varphi) \nabla \varphi\right\|_{L_{2}(Q)}=\|\bar{g}(u, \varphi) \nabla \ln (1-\varphi+\delta)\|_{L_{2}(Q)} \leqslant c_{1}\|\ln (1-\varphi+\delta)\|_{L_{2}(Q)} \leqslant c_{2} .
$$

Hence we obtain the a priori estimate

$$
\|u\|_{L_{\infty}\left(J ; L_{2}(\Omega)\right)}^{2}+\|\nabla u\|_{L_{2}(J \times \Omega)}^{2} \leqslant c
$$


with constant independent of $\delta$. Moreover, a standard dual estimate for equation (64) with the help of 73 shows

$$
\left\|\partial_{t} u\right\|_{L_{2}\left(J ; H^{1}(\Omega)^{*}\right)} \leqslant c
$$

and then by interpolation with (74),

$$
\|u\|_{H^{1 / 2}\left(J ; L_{2}(\Omega)\right)} \leqslant c .
$$

As a consequence there exists a sequence of approximation parameters $\delta_{k} \rightarrow 0$ and corresponding solutions $u_{k}=u_{\delta_{k}}, \varphi_{k}=\varphi_{\delta_{k}}$ such that $u_{k} \rightarrow u$ weakly in $H^{1 / 2,1}(Q) \cap H^{1}\left(J ; H^{1}(\Omega)^{*}\right)$ and, due to the compact embedding (68), strongly in $L_{r}(Q)$ for an arbitrarily chosen $r<2+4 / d, \varphi_{k} \rightarrow \varphi$ weakly in $H^{1}(Q)$ and strongly in $L_{s}(Q)$ for an arbitrarily chosen $s<+\infty$, and $\nabla \ln \left(1-\varphi_{k}+\delta_{k}\right) \rightarrow$ $\Theta$ weakly in $L_{2}(Q)$. As a consequence of the strong convergence of $\varphi_{k}$ and $u_{k}$ in $L_{s}(Q)$ and $L_{r}(Q)$ we also have convergence almost everywhere in $Q$ after extraction of a suitable subsequence. We pass to the limit $k \rightarrow 0$ and conclude that $(u, \varphi)$ is a solution to the problem

$$
\begin{aligned}
& \left\langle\partial_{t} u, v\right\rangle+\int_{Q} D(\nabla u-\bar{g}(u, \varphi) \Theta) \cdot \nabla v \mathrm{~d} x \mathrm{~d} t=0 \\
& \left\langle\alpha \epsilon^{2} \partial_{t} \varphi, w\right\rangle+\int_{Q}\left(\epsilon^{2} \nabla \varphi \cdot \nabla w+\left(p^{\prime}(\varphi)+\epsilon \beta q(u, \varphi)\right) w\right) \mathrm{d} x \mathrm{~d} t=0
\end{aligned}
$$

for all $v, w \in L_{2}\left(J ; H^{1}(\Omega)\right)$. It remains to prove $\Theta=\nabla \ln (1-\varphi)$. We first show $\varphi<1$ almost everywhere. Let us assume that this is not the case, say $\varphi=1$ on some set of measure $\zeta>0$. By the Egorov theorem there is a set $Q_{\zeta}$ of measure $\left|Q_{\zeta}\right|>|Q|-\zeta / 2$ such that $\varphi_{k} \rightarrow \varphi$ uniformly on the set $Q_{\zeta}$. As a consequence there is a set $S_{\zeta}$ of measure $\zeta / 2$ such that $\varphi_{k} \rightarrow 1$ uniformly on $S_{\zeta}$. Then the limit $k \rightarrow \infty$ in the estimate

$$
c_{1} \geqslant\left\|\ln \left(1-\varphi_{k}+\delta_{k}\right)\right\|_{L_{1}(Q)} \geqslant\left\|\ln \left(1-\varphi_{k}+\delta_{k}\right)\right\|_{L_{1}\left(S_{\eta}\right)}
$$

gives a contradiction, and therefore it is proved that $\varphi<1$. Now we consider the limit $k \rightarrow \infty$ in

$$
\int_{Q} \nabla \ln \left(1-\varphi_{k}+\delta_{k}\right) w \mathrm{~d} x \mathrm{~d} t=-\int_{Q} \ln \left(1-\varphi_{k}+\delta_{k}\right) \nabla \cdot w \mathrm{~d} x \mathrm{~d} t
$$

with some test function $w \in C_{0}^{\infty}\left(Q ; \mathbb{R}^{n}\right)$. The left hand side here converges to $\int_{Q} \Theta \cdot w \mathrm{~d} x \mathrm{~d} t$. Therefore it suffices to prove that the right hand side converges to $-\int_{Q} \ln (1-\varphi) \nabla \cdot w \mathrm{~d} x \mathrm{~d} t$. Consider some arbitrary positive $\eta$, with $\eta<|Q|$. Since $|\{(t, x) \in Q \mid \varphi(t, x)<1-\lambda\}|$ converges to $|\{(t, x) \in Q \mid \varphi(t, x) \leqslant 1\}|=|Q|$ as $\lambda \rightarrow 0$, there is some $\lambda=\lambda(\eta)>0$ such that $\varphi<1-\lambda$ on a set of measure $|Q|-\eta / 2$. Moreover, due to the Egorov theorem, $\varphi_{k}$ converges uniformly to $\varphi$ on a set of measure $|Q|-\eta / 2$. As a consequence we have $\varphi_{k} \rightarrow \varphi<1-\lambda$ on a set $R_{\eta}$ of measure $|Q|-\eta$. Then it follows that

$$
\int_{R_{\eta}} \ln \left(1-\varphi_{k}+\delta_{k}\right) \nabla \cdot w \mathrm{~d} x \mathrm{~d} t \rightarrow \int_{R_{\eta}} \ln (1-\varphi) \nabla \cdot w \mathrm{~d} x \mathrm{~d} t
$$

and, with $S_{\eta}:=Q \backslash R_{\eta}$,

$$
\left|\int_{S_{\eta}} \ln \left(1-\varphi_{k}+\delta_{k}\right) \nabla \cdot w \mathrm{~d} x \mathrm{~d} t\right| \leqslant\|\nabla \cdot w\|_{L_{\infty}(Q)}\left\|\ln \left(1-\varphi_{k}+\delta_{k}\right)\right\|_{L_{2}\left(S_{\eta}\right)}\left|S_{\eta}\right|^{1 / 2} \leqslant c \sqrt{\eta}
$$


with some constant $c$ independent of $\eta$. Moreover, due to $\varphi<1$ we have $\left|\ln \left(1-\varphi_{k}+\delta_{k}\right)\right| \rightarrow$ $|\ln (1-\varphi)|$ a.e. Using the Fatou Lemma we obtain

$$
\begin{aligned}
& \left|\int_{S_{\eta}} \ln (1-\varphi) \mathrm{d} x\right| \leqslant \int_{S_{\eta}}|\ln (1-\varphi)| \mathrm{d} x=\int_{S_{\eta}} \lim _{k \rightarrow+\infty}\left|\ln \left(1-\varphi_{k}+\delta_{k}\right)\right| \mathrm{d} x \\
& \quad \leqslant \liminf _{k \rightarrow+\infty} \int_{S_{\eta}}\left|\ln \left(1-\varphi_{k}+\delta_{k}\right)\right| \mathrm{d} x \leqslant \liminf _{k \rightarrow+\infty}\left\|\ln \left(1-\varphi_{k}+\delta_{k}\right)\right\|_{L_{2}(Q)}\left|S_{\eta}\right|^{1 / 2} \leqslant c \sqrt{\eta} .
\end{aligned}
$$

Since $\eta \in(0,|Q|)$ can be arbitrarily chosen, by letting $\eta \rightarrow 0$ we obtain

$$
\int_{Q} \ln \left(1-\varphi_{k}+\delta_{k}\right) \nabla \cdot w \mathrm{~d} x \mathrm{~d} t \rightarrow \int_{Q} \ln (1-\varphi) \nabla \cdot w \mathrm{~d} x \mathrm{~d} t .
$$

Hence the following theorem is proved:

THEOREM 2 Let $\alpha, \xi, D$ be positive constants, let $\Omega$ be a bounded $C^{2}$ domain of dimension $d \in\{2,3\}$, let the functions $p, q$ and $g$ satisfy the conditions 62 , 637, 669, (70) with Lipschitz functions $\bar{p}, \bar{q}$ and $\bar{g}$ that are bounded for $\varphi \in[-1,1]$ and $u \in \mathbb{R}$. Let the initial conditions satisfy $u_{0} \in L_{2}(\Omega), \varphi_{0} \in W_{s}^{2-2 / s}(\Omega)$ with $s>1+d / 2,-1<\varphi_{0}<1$ and $\ln \left(1-\varphi_{0}\right) \in H^{1}(\Omega)$. Then problem (64), (65) has a solution.

REMARK The condition in (70 $)$ is again satisfied by both the double well potentials (13) and (14). For the condition $(702)$ to hold for $q(u, \varphi)$ as in (58) and $k(\varphi)$ as in (15) it is again required that $f(u)-(\rho-u) f^{\prime}(u)$ is bounded. This may be achieved by a particular choice of $f(u)$ or, as mentioned before, by replacing the term by a bounded version as in 617.

\section{Numerical examples}

\subsection{ID example}

In this section we investigate numerically how well the phase field model approximates the sharp interface model. We first study the following 1D free boundary problem:

$$
\begin{cases}\partial_{t} u=\partial_{x}^{2} u & \text { for } x \in(-1, s(t)), \\ \partial_{x} u=0 & \text { for } x=-1, \\ \partial_{x} u=\dot{s}(\rho-u) & \text { for } x=s(t) \\ \dot{s}=-\left(u(t, s(t))-u^{*}\right), & \\ u(0, x)=u_{I}(x), & \\ s(0)=0 & \end{cases}
$$

with $\rho=1, u^{*}=0.5$ and $u_{I} \equiv 0.8$. We compute the solution to this free-boundary problem using the Arbitrary Lagrangian-Eulerian (ALE) method [8] with a discretization on 960 quadratic finite elements. We use this solution as the reference solution.

Substituting the form of $f(u)$ (which is $f(u)=u-u^{*}$ ) into 56 with $\alpha=1$, and using the 1D setting, we obtain the equations 


$$
\begin{cases}\epsilon^{2} \partial_{t} \varphi-\epsilon^{2} \partial_{x}^{2} \varphi+p^{\prime}(\varphi)+\epsilon \frac{16}{15} k^{\prime}(\varphi)(2 u-1.5)=0 & \text { for } x \in(-1,1), \\ \partial_{t} u-\partial_{x}^{2} u-\partial_{x}\left(\frac{(\rho-u) k^{\prime}(\varphi)}{k(\varphi)+\epsilon} \partial_{x} \varphi\right)=0 & \text { for } x \in(-1,1), \\ \partial_{x} \varphi=0 & \text { for } x \in\{-1,1\}, \\ \partial_{x} u=0 & \text { for } x \in\{-1,1\}, \\ \varphi(0, x)=\varphi_{I}(x), & \\ u(0, x)=u_{I}(x), & \end{cases}
$$

where the initial data are given by

$$
\varphi_{I}(x)=\left\{\begin{array}{ll}
-1 & \text { for } x \in[-1,0), \\
1 & \text { for } x \in[0,1],
\end{array} \quad u_{I}(x)= \begin{cases}0.8 & \text { for } x \in[-1,0), \\
1 & \text { for } x \in[0,1] .\end{cases}\right.
$$

We solve the phase field model for three different values of $\epsilon$, using a discretization on 1920 quadratic finite elements. The time discretization for both the sharp interface model and the phase field model is performed using variable step size, variable order BDF formulae with a relative and absolute error tolerance set to $10^{-7}$.

Notice that we use a coarser discretization in the ALE method for the reference solution of the sharp interface model than for computations for the phase field model. This may seem contradictory, but it is not: this is due to the different methods that are used. For the phase field model we need to resolve the steep transition of the phase parameter $\varphi$ at the position of the moving fluid/solid interface. Here we use a fixed, uniform discretization, so that we need quite a fine mesh, especially for small $\epsilon$. If an adaptive grid were used, fewer finite elements would have been sufficient. The ALE method for the sharp interface model does not need to resolve steep transitions. Instead, the movement of the free boundary is captured by moving the nodes along with the boundary. This means that for the ALE method sufficient accuracy can be obtained with a coarser grid.

In Figure 1 we plot the position of the free boundary $s(t)$ for the reference solution and the position of the zero level set of the phase field variable $\varphi(t, x)$. The sharp interface problem has a steady state for $s(t)=-0.6$ and $u(t, x)=u^{*}=0.5$ on $[-1,-0.6]$. We see that the reference

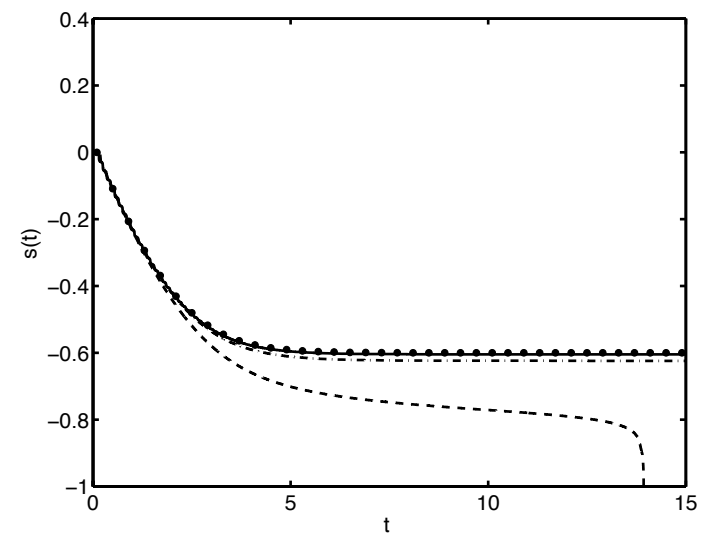

FIG. 1. The position of the free boundary $s(t)$ against time $t$. Dots: reference solution computed with the ALE method; dashed line: phase field model with $\epsilon=0.1$; dash-dotted line: $\epsilon=0.02$; solid line: $\epsilon=0.004$. 
solution approaches that solution asymptotically. The phase field model with $\epsilon=0.1$ does not approach a stationary solution, probably because of boundary effects, but this is mainly due to the regularization of the $u$-equation. If we compute the solution, with $\epsilon=0.1$ in the phase field equation, and $\epsilon=0.001$ in the $u$-equation we obtain a solution that is in the plot indistinguishable from the reference solution and the solution with $\epsilon=0.004$ in the phase field equation.

\subsection{2 example}

For a $2 \mathrm{D}$ test problem we solve the equations

$$
\begin{cases}\partial_{t} u=\Delta u & \text { for } x \in \Omega_{f}(t), \\ \nabla u \cdot n=0 & \text { for } x \in \Gamma_{f}, \\ \nabla u \cdot n=v_{n}(\rho-u) & \text { for } x \in \Gamma_{I}(t), \\ v_{n}=-\left(u-u^{*}\right)-\sigma \kappa & \text { for } x \in \Gamma_{I}(t), \\ u(0, x)=0 & \text { for } x \in \Omega_{f}(0)\end{cases}
$$

with $\rho=1, \sigma=0.1$ and $u^{*}=0.5$. The initial domain $\Omega_{f}(0)$ is depicted in Figure 2 We compute the solution to this free-boundary problem using the ALE method with a discretization on 52608 quadratic finite elements. We use this solution as the reference solution.

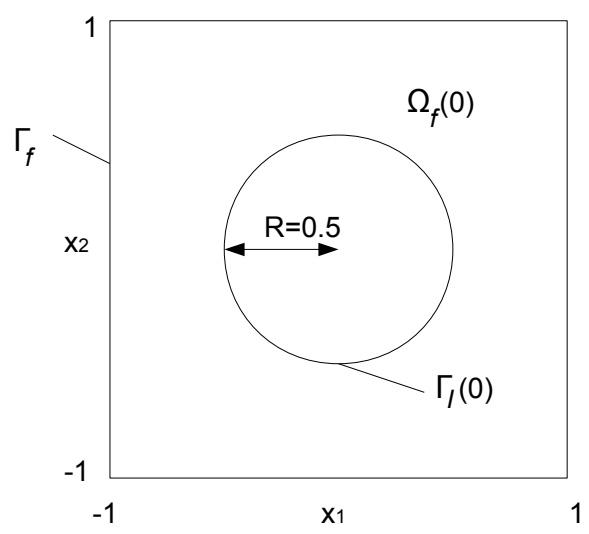

FIG. 2. The initial configuration for the sharp interface model.

We approximate the reference solution using the following phase field model:

$$
\begin{aligned}
\alpha \epsilon^{2} \partial_{t} \varphi-\epsilon^{2} \Delta \varphi+p^{\prime}(\varphi)+\epsilon \beta k^{\prime}(\varphi)\left(f(u)+(u-\rho) f^{\prime}(u)\right) & =0, \\
\partial_{t} u-\nabla \cdot\left(\nabla u+(\rho-u) \frac{k^{\prime}(\varphi)}{k(\varphi)+\delta} \nabla \varphi\right) & =0,
\end{aligned}
$$

on the time-space cylinder $Q(T):=J \times \Omega$ with time interval $J:=(0,0.6]$, where the domain $\Omega$ is given by the square $\Omega=[-1,1]^{2}$. On $\partial \Omega$ we have for both $\varphi$ and $u$ homogeneous Neumann boundary conditions: $\nabla \varphi \cdot n=0$ and $\nabla u \cdot n=0$. We use the following parameter values:

$$
\alpha=10, \quad \epsilon=0.01, \quad \beta=32 / 3, \quad f(u)=u-u^{*}, \quad \rho=1, \quad u^{*}=0.5, \quad \delta=0.001 .
$$



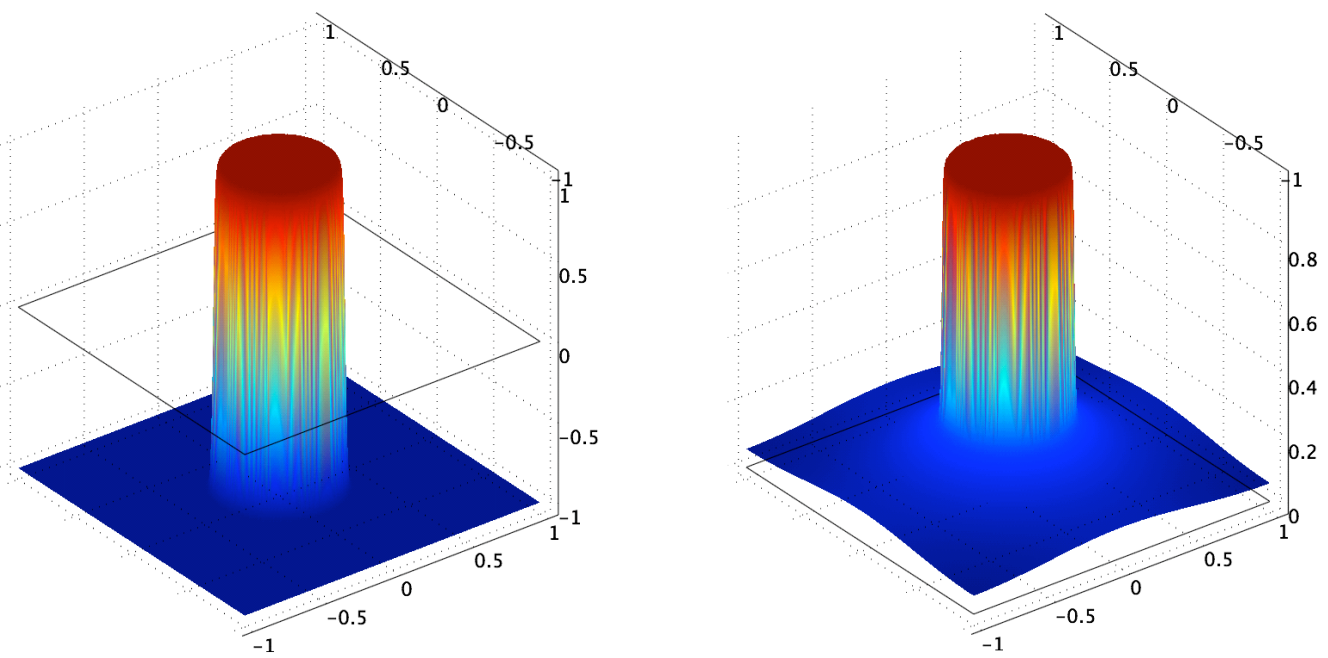

FIG. 3. Plots of the phase field $\varphi$ (left) and the concentration $u$ (right) for $t=0.25$.

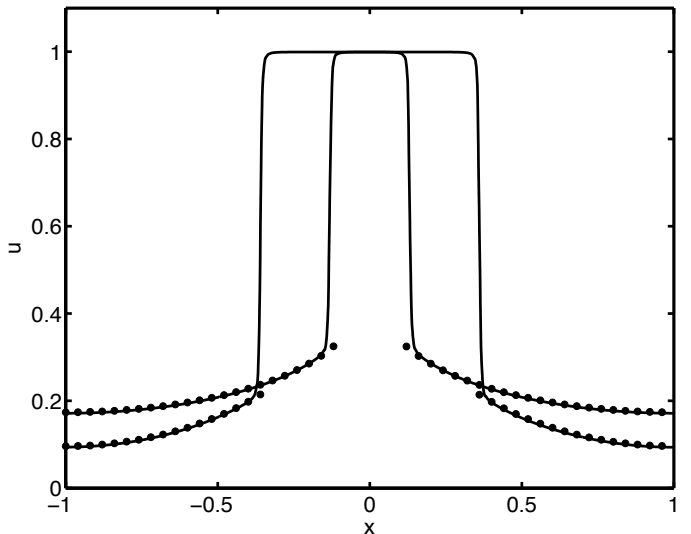

FIG. 4. Profiles of $u$ along the line $x_{2}=0$ for $t=0.25$ and $t=0.6$. Dots: reference solution computed with the ALE method; solid line: phase field model.

The initial data are given by

$$
\varphi_{I}(x)=\left\{\begin{array}{ll}
-1 & \text { for } x \in[-1,1]^{2},|x| \geqslant 1 / 2, \\
1 & \text { for } x \in[-1,1]^{2},|x|<1 / 2,
\end{array} \quad u_{I}(x)= \begin{cases}0 & \text { for } x \in[-1,1]^{2},|x| \geqslant 1 / 2, \\
1 & \text { for } x \in[-1,1]^{2},|x|<1 / 2 .\end{cases}\right.
$$

We solve the phase field model using a discretization on 62720 quadratic finite elements. The time discretization for both the sharp interface model and the phase field model is performed using variable step size, variable order BDF formulae with a relative and absolute error tolerance set to $10^{-4}$. In Figure 3 , we show the solution to the phase field model for $t=0.25$. We see that a part of the initial crystalline solid has dissolved, and that the crystalline solid is still more or less circular 
in shape. In order to compare the solution to the phase field model with the reference solution, we plot in Figure 4 the profiles of the concentration $u$ for both the reference solution and the phase field solution for two time instants, $t=0.25$ and $t=0.6$, along the cross section on the line $x_{2}=0$. We see that the solutions agree very well on the fluid domain.

\subsection{Convergence}

In this section we show a more quantitative comparison of the phase field model and the sharp interface model. We consider the phase field model

$$
\begin{aligned}
\alpha \epsilon^{2} \partial_{t} \varphi-\epsilon^{2} \partial_{x}^{2} \varphi+p^{\prime}(\varphi)+\epsilon \beta k^{\prime}(\varphi)\left(f(u)+(u-\rho) f^{\prime}(u)\right) & =0, \\
\partial_{t} u-D \partial_{x}\left(\partial_{x} u+(\rho-u) \frac{k^{\prime}(\varphi)}{k(\varphi)+\delta} \partial_{x} \varphi\right) & =0
\end{aligned}
$$

on the interval $[-0.01,0.01]$ with the parameter values

$$
\alpha=1, \quad D=10^{-5}, \quad \beta=16 / 15, \quad f(u)=0.01\left(u-u^{*}\right), \quad \rho=1, \quad u^{*}=0.5,
$$

and varying values of $\epsilon$ and $\delta$. We compare solutions to this phase field model with solutions to the free boundary problem given by

$$
\begin{cases}\partial_{t} u=D \partial_{x}^{2} u & \text { for } x \in(-0.01, s(t)), \\ \partial_{x} u=0 & \text { for } x=-0.01, \\ \partial_{x} u=\dot{s}(\rho-u) & \text { for } x=s(t), \\ \dot{s}(t)=-0.01\left(u(t, s(t))-u^{*}\right), & \\ u(0, x)=u_{I}(x), & \\ s(0)=0 & \end{cases}
$$

with $u_{I} \equiv 0.8$.

In order to study the convergence of the phase field model, we show the absolute value of the difference between the computed position of the interface-defined as zero point of the phase field-at time $t=1$ and the position of the interface as given by the reference solution to the corresponding sharp interface model computed using the ALE method for several selected discretization parameters. The reference solution is computed using a grid consisting of 1920 elements. The interface position (for the sharp interface model) as given by the reference solution is $s(1)=-0.001867$.

In Table 1 the difference between the position of the interface computed using the phase field model and the position of the interface in the ALE reference solution at $t=1$ is given. For $\epsilon=$ 0.0005 the phase field model does not give a very accurate prediction of the position of the interface: the relative error (error divided by the interface position) is of order one. In this case the value of $\delta$ and the number of elements used do not improve the accuracy of the phase field model very much. For smaller values of $\epsilon$ the accuracy of the phase field model improves, but also here the value of $\delta$ and the number of elements improve the accuracy very little. The results, however, indicate that the phase field model with small values of $\epsilon$ approximates the behaviour of the sharp interface model quite accurately. 
TABLE 1

Comparison results

\begin{tabular}{rrrrr}
\hline$\epsilon$ & $\delta$ & \# elem. & error & rel. error \\
\hline 0.0005 & 0.0005 & 480 & $1.5739 \mathrm{e}-3$ & $8.4299 \mathrm{e}-1$ \\
0.0005 & 0.0001 & 480 & $1.5690 \mathrm{e}-3$ & $8.4041 \mathrm{e}-1$ \\
0.0005 & 0.00002 & 480 & $1.5679 \mathrm{e}-3$ & $8.3981 \mathrm{e}-1$ \\
0.0005 & 0.0005 & 1920 & $1.5710 \mathrm{e}-3$ & $8.4147 \mathrm{e}-1$ \\
0.0005 & 0.0001 & 1920 & $1.5659 \mathrm{e}-3$ & $8.3872 \mathrm{e}-1$ \\
0.0005 & 0.00002 & 1920 & $1.5654 \mathrm{e}-3$ & $8.3847 \mathrm{e}-1$ \\
0.0005 & 0.0005 & 7680 & $1.5712 \mathrm{e}-3$ & $8.4158 \mathrm{e}-1$ \\
0.0005 & 0.0001 & 7680 & $1.5664 \mathrm{e}-3$ & $8.3900 \mathrm{e}-1$ \\
0.0005 & 0.00002 & 7680 & $1.5654 \mathrm{e}-3$ & $8.3844 \mathrm{e}-1$ \\
0.0001 & 0.0005 & 1920 & $2.0733 \mathrm{e}-5$ & $1.1105 \mathrm{e}-2$ \\
0.0001 & 0.0001 & 1920 & $2.0121 \mathrm{e}-5$ & $1.0777 \mathrm{e}-2$ \\
0.0001 & 0.00002 & 1920 & $1.9812 \mathrm{e}-5$ & $1.0612 \mathrm{e}-2$ \\
0.0001 & 0.0005 & 7680 & $1.8981 \mathrm{e}-5$ & $1.0166 \mathrm{e}-2$ \\
0.0001 & 0.0001 & 7680 & $1.8234 \mathrm{e}-5$ & $9.7666 \mathrm{e}-3$ \\
0.0001 & 0.00002 & 7680 & $1.7974 \mathrm{e}-5$ & $9.6274 \mathrm{e}-3$ \\
0.00002 & 0.0005 & 7680 & $5.1131 \mathrm{e}-6$ & $2.7387 \mathrm{e}-3$ \\
0.00002 & 0.0001 & 7680 & $4.6235 \mathrm{e}-6$ & $2.4765 \mathrm{e}-3$ \\
0.00002 & 0.00002 & 7680 & $4.4945 \mathrm{e}-6$ & $2.4074 \mathrm{e}-3$ \\
\hline
\end{tabular}

\section{Conclusions}

We have proposed a phase field model for a precipitation/dissolution process. It describes a one-phase free boundary problem with a kinetic condition at the moving boundary. In addition, instead of the jump of the concentration across the interface, the concentration on one side of the interface is prescribed, and the normal velocity of the interface depends nonlinearly on the variable concentration on the other side of the interface. Using a formal asymptotic analysis we have shown that the phase field model approximates in the limit $\epsilon \rightarrow 0$ the appropriate sharp interface model. Here $\epsilon$ is the thickness of an interfacial layer containing the moving boundary. The existence of a solution to the phase field model has been proved for the cases of a nonsingular and a singular coefficient. Uniqueness of the solution is established for the nonsingular version only. The convergence behaviour of the phase field model to the sharp interface model is supported by numerical evidence.

\section{Acknowledgments}

The authors would like to thank I. S. Pop and A. Muntean for the discussions that helped to shape the work in this paper. The second author would like to thank the Stuttgart Research Center "Simulation Technology" for supporting this research.

\section{REFERENCES}

1. Bergh, J., \& Löfström, J. Interpolation Spaces. An Introduction. Grundlehren Math. Wiss. 223, Springer, Berlin (1976). Zbl 0344.46071 MR 0482275

2. Caginalp, G. An analysis of a phase field model of a free boundary. Arch. Ration. Mech. Anal. 92 (1986), 205-245. Zbl 0608.35080 MR 0816623 
3. Caginalp, G., \& Chen, X. Convergence of the phase field model to its sharp interface limits. Eur. J. Appl. Math. 9 (1998), 417-445. Zbl 0930.35024 MR 1643668

4. Caginalp, G., Chen, X., \& Eck, C. A rapidly converging phase field model. Discrete Contin. Dynam. Systems 15 (2006), 1017-1034. Zbl 1119.80009

5. Caginalp, G., \& Fife, P. C. Dynamics of layered interfaces arising from phase boundaries. SIAM J. Appl. Math. 48 (1988), 506-518. MR 0941098

6. Caginalp, G., \& Jones, J. A derivation and analysis of phase field models of thermal alloys. Ann. Phys. 237 (1995), 66-107.

7. Caginalp, G., \& XIE, W. An analysis of phase-field alloys and transition layers. Arch. Ration. Mech. Anal. 142 (1998), 293-329. Zbl 0906.73008 MR 1645959

8. Donea, J., Huerta, A., Ponthot, J.-Ph., \& Rodríguez-Ferran, A. Arbitrary LagrangianEulerian methods. In: Encyclopedia of Computational Mechanics, Volume 1: Fundamentals, Chap. 14, E. Stein et al. (eds.), Wiley (2004).

9. ECK, C. Homogenization of a phase field model for binary mixtures. Multiscale Model. Simul. 3 (2004), 1-27. Zbl 1160.35332 MR 2123107

10. FASANO, A. Mathematical models of some diffusive processes with free boundaries. MAT. Serie A: Conferencias, Seminarios y Trabajos de Matemática 11, Universidad Austral, Facultad de Ciencias Empresariales (FCE-UA), Departamento de Matemática, Rosario (2005). Zbl 1122.35001 MR 2285006

11. Knabner, P., van Duijn, C. J., \& Hengst, S. An analysis of crystal dissolution fronts in flows through porous media. Part 1: Compatible boundary conditions. Adv. Water Res. 18 (1995), 171-185.

12. Solonnikov, V. A. On boundary value problems for linear parabolic systems of differential equations of general form. Trudy Mat. Inst. Steklov. 83 (1965), 3-163 (in Russian); English transl.: Proc. Steklov Inst. Math. 83 (1967), 1-184. Zbl 0164.12502 MR 0211083

13. VAN DUIJN, C. J., \& POP, I. S. Crystal dissolution and precipitation in porous media: pore scale analysis. J. Reine Angew. Math. 577 (2004), 171-211. Zbl 1060.76116 MR 2108218

14. VAN Noorden, T. L. Crystal precipitation and dissolution in a porous medium: Effective equations and numerical experiments. Multiscale Model. Simul. 7 (2009), 1220-1236. Zbl 1176.35020 MR 2480118

15. van Noorden, T. L. Crystal precipitation and dissolution in a thin strip. Eur. J. Appl. Math. 20 (2009), 69-91. Zbl 1154.82321 MR 2471668

16. VAN NoORden, T. L., \& POP, I. S. A Stefan problem modelling crystal dissolution and precipitation. IMA J. Appl. Math. 73 (2008), 393-411. Zbl 1153.80004 MR 2411490 